NBER WORKING PAPER SERIES

\title{
MODERATING ELECTIONS
}

\author{
Alberto Alesina \\ Howard Rosenthal
}

Working Paper No. 3072

\section{NATIONAL BUREAU OF ECONOMIC RESEARCH \\ 1050 Massachusetts Avenue \\ Cambridge, MA 02138 \\ August 1989}

This work was supported by National Science Foundation grant SES-8821441. This paper is part of NBER's research program in Financial Markets and Monetary Economics. Any opinions expressed are those of the authors not those of the National Bureau of Economic Research. 


\section{NBER Working Paper \#3072 \\ August 1989}

\section{MODERATION ELECTIONS}

\section{ABSTRACT}

This paper extends the spatial theory of voting to an institutional structure in which policy cholces are a function of the composition of the legislature and of the executive. In an institutional setup in which the policy outcome depends upon relative plurality, each voter has incentives to be strategic since the outcome depends upon how everybody else votes. By applying to this game between voters the refinements of Strong Nash and Coalition Proof Nash we prove existence of equilibria with properties which appear intuitive and realistic. In fact, the model has several testable implications which seem consistent with some observed patterns of voting behavior in the United States and perhaps in other democracies in which the executive is directly elected. For instance, the model predicts: a) split-ticket voting; b) for some parameter values, a split government with different parties controlling the executive and the majority of the legislature; and c) the mid-term electoral cycle.

\author{
Alberto Alesina \\ Harvard University \\ Department of Economics \\ Cambridge, MA 02138
}

Howard Rosentha1

Carnegie-Me11on University

Department of Economics

Pittsburgh, PA 15213 


\section{INTRODUCTION}

Policy outcomes typically reflect the composition of both the executive and the legislature. In the United States, for instance, the administration's influence on policy making is affected by the composition of Congress.

Traditional spatial models of elections ignore this institutional complexity and assume that the winner of a two-party race has full control over policy. ${ }^{1}$ These models focus on the strategic interaction between the candidates and treat each voter as a passive player, voting for the candidate offering the policy closest to his ideal point.

In our model the voters instead face two parties with policy-preferences [Wittman (1977, 1988)]; that is, party preferences are defined on policy outcomes. Without "checks" by the legislature, executives representing the two parties would follow distinct and polarized policies. ${ }^{2}$ Voters with ideal policies intermediate between the ldeal policies of the two parties take advantage of the institutional structure to "moderate" the executive and thus achieve a pollicy outcome closer to their ideal. In fact, the actual policy not only depends upon the identity of the executive but also reflects the relative plurality of the two parties in the legislature. This realistic feature of the model implies that, unlike in the traditional spatial model, the decision of each voter depends upon his beliefs about the behavior of other voters. Indeed, one needs to consider strategic voting behavior whenever policy reflects more than a single binary cnolce. Thus, we consider a coordination game between the voters in the context of the "new institutionalism's" concern about the Interaction of the executive and the legislature [Shepsle (1986), Hammond and Miller (1988)]. 
Our model of "moderating behavior" has implicatlons which are consistent with some observed voting patterns in the United States. For instance, when the executive and the legislature are elected simultaneously, we predict that split-ticket voting generally occurs. That is, some voters vote for different parties in the two elections. Consequently, the party winning the election for the executive branch may not have a majority in the legislature. In fact, for some parameter values the model predicts divided government, with different parties controlling the executive and the legislature. ${ }^{3}$

Second, the model is consistent with a persistent phenomenon in the United States: the "mid-term voting cycle". In mid-term congressional elections, the party holding the executive loses plurality relative to the preceeding congressional elections held simultaneously with the presidential election. Even though other explanations of this strong regularity have been proposed, such as the "coattatl effect", we offer a different explanation which abstracts from personal characteristics of presidential candidates, incumbency preferences, etc. An application of this model to macroeconomic policy has been tested in Alesina and Rosenthal (1989); this "moderating" model performs at least as well and often better than traditional empirical voting models based upon retrospective voting behavior and incumbency advantage.

We investigate both a full information and an incomplete information model. In the former, but not in the latter, the distribution of voters' Ideal points is common knowledge. Some of the results concerning split-ticket voting and moderating behavior emerge in both cases and can be more easily illustrated in the certainty model, which can be developed with rather general assumptions about utility functions, distributions of ideal 
points, and the impact of the legislative plurality on policy. A mid-term cycle emerges only in the incomplete information model. Explicit solutions for this case can be obtained under more restrictive functional forms, which are needed for computational reasons. Small deviations from these assumptions should result in small quantitative differences and no qualitative differences in the results.

From a technical point of view, this paper shows how the Strong Nash (SN) [Aumann (1959)] and Coalition Proof Nash Equilibria (CPN) [Bernheim, Peleg and Whinston (1987)] refinements can be usefully applied to voting games. Since we consider a continuum of voters, and the CPN concept is defined only for a finite number of players, we use Greenberg's (1989) results to develop a directly analogous refinement for the continuum case. This refinement is a Von Neuman Morgenstern (vN\&M) stable set.

These refinements are needed for two reasons. First, with atomistic voters, every strategy is Nash for the individual voter, since he does not have any measurable impact on the outcome. When legislative elections are held separately, at the "mid term" of a presidential term, both the SN and the VN\&M refinements lead to a unique equilibrium that is directly analogous to the unique Nash equilibrium with a finite number of voters. This SN-vN\&M equilibrium has the intuitive property that no voter would like to see a decline in the plurality of the party he is voting for.

Second, with elther a finite or an infinite number of voters, the "all or none" feature of the majority rule used in presidential elections requires refinements beyond the simple Nash notion. We need refinements to address the situation where no voter is pivotal in the presidential election.

In the specific context of our certainty model, when the president is 
elected simultaneously with the legislature, there are two possible equilibria: one with party $D$ winning the presidency and a legislative vote identical to the SN outcome when party " $D$ " holds the executive and legislative elections are held separately, as in the "midterm", and another corresponding to the outcome when party " $R$ " holds the executive when midterm elections occur. ${ }^{5}$ We need refinements to indicate whether one or both of the two outcomes is "stable". It turns out that SN is too strong a refinement. We show that, for some parameter values, there is no SN equilibrium when the president and legislature are elected simultaneously. In contrast, a vN\&M always exists although, when the two parties are not too asymmetrically located with respect to the median voter, both outcomes may be vN\&M. Again, the refinements appear to match intuition. For example, when both parties are to the same side of the median voter, there is a unique SN-vN\&M where the party closer to the median holds both the presidency and a majority in the legislature. Moreover, for symmetric utility and symmetric distributions of voter ideal points, the outcome where the party closer to the median wins the presidency is always vN\&M.

Fiorina (1988) has recently proposed a model of split-ticket voting which is related to ours. There are three important differences between the models. Fiorina's specification of executive-legislature interaction is such that only four policy outcomes are possible, corresponding to the four combinations of parties holding the executive and the majority in the legislature, while we allow for a continuum of policies. Second, and more importantly, Fiorina does not consider strategic voting. Third, since Fiorina considers only a one period model, he does not address the relationship between split-tickets and the mid-term cycle. 


\section{THE MODEL WITH COMPLETE INFORMATION}

To facilitate intultion, we first present a simple version of the model. In Section 3 several restrictive assumptions are relaxed.

\subsection{Assumptions and Institutional Setup}

We consider two-party systems in which the parties (identified with the candidates) have policy preferences. In particular, we assume that the two parties and the voters have unldimensional, continuous, single peaked and strictly concave utility functions over a single policy issue. There are two periods and utility functions are intertemporally additive with discount factor $\beta \leq 1$. All the utility functions are identical except for a bliss point. In each period $t, t=1,2$, the two parties, labelled $D$ and $R$, have objectives written as:

$$
u_{t}=u\left(x_{t}, \theta_{j}\right) \quad J=D, R
$$

where $x$ represents the policy issue, $\theta_{D}$ and $\theta_{R}$ are the two bliss points, with $\theta_{D}<\theta_{R}$. The population of voters is infinite, and each voter, indexed by $i$, has an objective function analogous to (1), with bliss point $\theta_{i}$. The distribution of voters' bliss points is uniform and normalized in the closed interval $I=[0,1]$. We also impose the unrestrictive assumption $0<\theta_{D}<\theta_{R}<$ 1.

In the complete information model the preferences of both parties and voters are common knowledge. Thus, if the party in office has complete control of policy, it follows its ideal policy $\left(\theta_{D}\right.$ or $\left.\theta_{R}\right)$. This is the only sub-game perfect equilibrium in a finitely repeated electoral game as shown in Alesina (1988). In fact, no other policy announced prior to elections would be implemented by the elected party, given that the party can choose Its ideal policy when in office. Thus, rational voters would not believe in 
any announcement other than the party ideal policy.

Our model of institutional structure posits that policy is determined by a "compromise" between the preferences of the president and those of the legislature. Let us define $V_{J t}, J=D, R$, to be the proportion of votes obtained by party $j$ in the legislative elections. Time subscripts are dropped where there is no possibility of confusion. Since we assume full turnout, $V_{R}=1-V_{D}$. When $D$ is president, the actual policy, $x^{D}$, is as follows:

$$
x^{B}=\theta_{D}+k V_{R}
$$

When $R$ holds the presidency, the policy $x^{R}$, is given by:

$$
x^{R}=\theta_{R}-k V_{D}=\theta_{R}-k\left(1-V_{R}\right)
$$

We also impose the condition

$$
0 \leq k<\theta_{R}-\theta_{D}
$$

which implies that the policy outcomes are in the interval $\left[\theta_{D}, \theta_{R}\right]$ and that $x^{D}<x^{R}$ for any $V_{R}{ }^{6}$ The legislature is elected by strict proportional representation in a single national district. The president is elected by majority rule.

We consider several electoral scenarios. The most general one is meant to mimic the United States case; in this scenario, at $t=1$, the executive and the legislature are elected contemporaneously; at $t=2$, there is another legislative vote, while the president elected at $t=1$ remains in office. That is, the president serves two periods while the legislature serves one period. ${ }^{7}$

Each voter moves in both periods. A voter can vote $D$ or $R$. Randomization is ruled out. The first period move is indicated as DD, RR, $\mathrm{DR}$, or $\mathrm{RD}$, where the first letter represents the presidential choice, the 
second the legislative choice. A single choice between $D$ or $R$ describes the move in the second perlod, when only the legislature is chosen. Thus, "RR in period $1, D$ in period 2 if $R$ president, $R$ in period 2 if $D$ president," lllustrates an example of a voter strategy.

Note that the vote shares of the first period elections are irrelevant for the second period elections. Who holds the presidency is the only relevant conditioning information for the second period component of voters strategies. This is true not only because there is no learning but also because the entire legislature is up for election at $t=2$. Policy at $t=2$ depends only upon who holds the presidency and not on the size of the legislative majority at $t=1$. The preceeding discussion, including the assumption that the party ideal policies are fixed, implies that any finite numbers of repetitions of this game can be captured with a two period analys is.

\subsection{Equilibrium: Definitions}

To focus on Nash equilibria that correspond to intuition, we consider the equilibrla which are in the core and/or in the Von Neumann Morgenstern abstract stable set of our game with the dominance relation proposed by Greenberg (1989), which we now define.

Let $z_{1}$ be an individual strategy, $z^{\prime}$ be $I^{\prime} s$ (nonempty and compact) strategy set, $Z^{M}$ the Cartesian product of the $Z^{i}$, and $z$ an element of $Z^{M}$, where $M$ denotes the set of players. Let $u_{1}(z)$ denote 1 's utility under $z$. $S$ and $S^{\prime}$ denote subsets of $M$.

Consider the abstract system $(W,<)$ :

$$
W \equiv\{(S, z) \mid S S M, S \neq \varnothing\}
$$

For $\left(S^{\prime}, z^{\prime}\right)$ and $(S, z)$ in $W$, 


$$
\begin{gathered}
\left(S^{\prime}, z^{\prime}\right)<(S, z) \Leftrightarrow S \subset S^{\prime}, u_{1}(z)>u_{1}\left(z^{\prime}\right) \text { for all } i \in S \\
\text { and } z_{1}^{\prime}=z_{1} \text { for all } 1 \notin S^{8}
\end{gathered}
$$

The core of the system is the set of undominated elements $B$,

$$
B=W \Delta(W)
$$

where $\Delta(a)=\{b \in W \mid b<a)$ and for some subset $K \subseteq W, \Delta(K)=U\{\Delta(a) \mid a \in K\}$.

Moreover, a set $\mathrm{K}$ is an $\underline{\text { vN\&M }}$ abstract stable set if $K=W \Delta(K)$. (That is, $K$ is internally and externally stable.)

This dominance relation is particularly appealing because Greenberg (1989) shows that for a game with a finlte number of players there exists a correspondence between the core and the set of SN equilibria and between the VN\&M stable set and the set of CPN equilibria." More specifically. Greenberg establishes the following result:

Theorem: For a finite set of players N, (i) the elements $z$ of the pairs $(N, z)$ that are in the abstract core $B$ represent the set of SN equilibria to the game defined by $Z^{N}$; (ii) $(N, z)$ appears as an element of an abstract stable set if and only if $z$ is in the set of CPN for the normal form of the game defined for $\mathrm{N}$.

Greenberg's proof is for a finite number of players; however the proof of part (i) carries through to the continuum trivially. The notion of CPN has not been defined for a continuum of players, since it is based upon a recursive argument. However the stable set defined with Greenberg's dominance relation extends the basic intuition of CPN to the continuum case. That is, an equilibrium is CPN if no coalition of players can credibly threaten to deviate from it by changing their strategies and improving their payoff. The requirement of credibility, that is the requirement that no sub-coalition could threaten to deviate from the original deviation, is what 
distingulshes CPN from SN. The importance of this distinction will become apparent when we study the simultaneous election of the president and the legislature.

It will be shown that equilibria in the stable set always yield policy outcomes equivalent to those brought about by a subset of the equilibria that can be described by cutpoint strategles, which we now define.

Definition: An element $z$ of $z^{t}$ is a cutpoint strategy for an electoral contest if there exists $\theta$ such that voters with $\theta_{1}<\theta$ vote $D$ and voters with $\theta_{1}>\theta$ vote $R$ in that contest. The vote of the individual of $\theta_{1}=\theta$ does not Influence the analysis because of zero measure.

We denote cutpoints in presidential contests by $\hat{\theta}$ and in legislative contests by $\tilde{\theta}$. We will show that leglslative strategles always follow a cutpoint rule. In presidential elections, instead non-cutpoint equilibrium strategies are possible, as a result of majority rule. Suppose, for example, that, conditional on the legislative components of strategies, a set of voters prefers the policy outcome associated with a $R$ presidential victory. If cutpoint strategles were followed these voters would vote $R$ for president. However, If these voters are not pivotal, they may vote $D$ for president without changing the pollcy outcome. Note that if these voters attributed even an infinitesimal possibility to being pivotal, they would vote $R$, thus Implying a cutpoint strategy. Consequently, a cutpoint ( $\hat{\theta})$ for presidential election is the most natural type of equilibrium even though we do not impose this restriction. This lack of restriction in the strategy space makes the statement of propositions and proofs significantly more laborious. 
Consequently, in the main text we consider only cutpoint strategies. This restriction is dropped in the Appendix. ${ }^{9}$

With cutpoint strategies, there are only three types of voters in the first period equilibrium. For instance, if $\hat{\theta}<\tilde{\theta}$ we have $D D$ voters who have ideal points below $\hat{\theta}, R D$ voters who have ideal points between $\hat{\theta}$ and $\tilde{\theta}$ and $R R$ voters who have ideal points above $\tilde{\theta}$.

\subsection{Equilibrium: Characterizations}

To illustrate critical aspects of the model, we consider several institutional scenarios, building up from the simplest one.

\section{3. a No Legislature}

With no legislative influence, $(k=0)$, the elected president determines policy for both periods. In this case, one can readily verify that a $S N$ equilibrium exists with cutpoint $\hat{\theta}$ implicitly defined by:

$$
u\left(\theta_{D}, \hat{\theta}\right)=u\left(\theta_{R}, \hat{\theta}\right)
$$

This is the standard result of the theory of two candidate elections. While if $\hat{\theta} \neq 1 / 2$ an infinity of other cutpoints also are $\mathrm{SN}, \hat{\theta}$ is a unique cutpoint under the assumption that no voter uses a weakly dominated strategy.

When preferences are symmetric, we obtain:

$$
\hat{\theta}=\frac{\theta_{R}+\theta_{D}}{2}
$$

As a benchmark, Figure $1 \mathrm{~A}$ indicates whether $D$ winning or $R$ winning is the SN outcome for $0 \leq \theta_{D} \leq 1 / 2 \leq \theta_{R} \leq 1$.

\section{3.b Legislative Elections: A Necessary Condition on Dominance}

Let $\mathrm{P}_{t}$ be the probability, immediately prior to period $t$ elections, that party $R$ holds the presidency in period $t$. Using (1), (2) and (3) the objective function of voter $i$ at $t=2$ is given by:

$$
u_{12}=P_{2} u\left(\theta_{R}-k\left(1-V_{R 2}\right), \theta_{1}\right)+\left(1-P_{2}\right) u\left(\theta_{D}+k V_{R 2}, \theta_{1}\right)
$$


where $P_{2}=1$ if president $R$ is in office or $P_{2}=0$ if $D$ is in office.

At $t=1$ the objective function of voter $i$ can be written as:

$$
u_{11}=\sum_{t=1}^{2} \beta^{t-1}\left[P_{1} u\left(\theta_{R}-k\left(1-V_{R t}\right), \theta_{1}\right)+\left(1-P_{1}\right) u\left(\theta_{D}+k V_{R t}, \theta_{1}\right)\right]
$$

In the complete information model $P_{1}$ can assume only three values: $P_{1}=1$ if the equilibrium strategies imply an $R$ victory in the presidential race, $P_{1}=0$ if they imply a $D$ victory, or $P_{1}=1 / 2$ in case of a tie. In the incomplete information model analyzed in Section $4, P_{1}$ can assume any value in $[0.1]$.

From (6) and the strict concavity of the function $u(\cdot)$, the following result follows immediately:

Lemma 1

For fixed $P_{t}$, a voter's indirect utility function on $V_{R t}$ is continuous and single-peaked.

Let us define $V_{R}^{1 P}$ as voter $I^{\prime}$ 's indirect bliss point for party $R^{\prime} s$ vote in the legislation election when the probability that $R$ wins the presidency is $P, V_{R}^{1 R}$ as the bliss point when the probability that $R$ wins the presidency is 1 , and $V_{R}^{1 D}$ as the bliss point when the probabllity that $R$ wins the presidency is 0 . We now state our central result concerning voting in legislative elections.

\section{Proposition 1}

In period $t$, for any value of $P_{t}, a$ necessary and sufficient condition for $(S, z)$ to be undominated by any $(T, y)$ where $y$ differs from $z$ only in the period $t$ legislative components is: 
for all measurable $T \subset S$ such that $i \in T$ votes $R$ in the legislative election, $V_{R}(z) \leq V_{R}^{1 P}$ and

(C)

for all measurable $L \subset S$ such that $i \in L$ votes $D$ in the legislative election, $V_{R}(z) \geq V_{R}^{1 P}$.

\section{Proof:}

If (C) is not satisfied, either (a) there is some TCS such that $i \in T$ votes $D$ and $V_{R}(z)<V_{R}^{i P}$ or $(b)$ some $L C S$ such that $i \in L$ votes $R$ and $V_{R}(z)>$ $V_{R}^{l P}$. Without loss of generality, assume (a). By continuity, there exists a measurable subset of voters $T^{\prime} C T$ whose utility would be increased if they switched the legislative component of their strategy $z_{1}$ to R. So an (S,z) that does not satisfy the condition is dominated. The proof of sufficiency is immediate.

Q.E. D.

This proposition implies that, in a SN equilibrium, there are no voters who would like to see a reduction of the share of votes of the party they are voting for in the legislative election.

2.3.c Legislative elections at $t=2$.

We now establish a result that resembles the median voter theorem. In traditional two party electoral models, policy equals the bliss point of the median voter. In our setup, we have a "pivotal voter theorem" where policy equals the bliss point of the pivotal voter in the legislative election. Let us define $\tilde{\theta}_{f}$ as the ldeal point of the cutpoint voter in the legislative elections when party $j$ holds the presidency.

Proposition 2: Pivotal Voter Theorem

The cutpoint strategy $\tilde{\theta}_{R}=\frac{\theta_{R}}{1+k}$ represents, the unique $S N$ and $v N \& M$ for president $R$ in office. 
The cutpoint strategy $\tilde{\theta}_{D}=\frac{\theta_{D}+k}{1+K}$ represents the unique $S N$ and vN\&M for president $D$ in of $f$ ice.

$$
\theta_{D}<\tilde{\theta}_{D}=x^{D}<\tilde{\theta}_{R}=x^{R}<\theta_{R}
$$

\section{Proof:}

$$
\begin{aligned}
& \text { For } v_{R}^{1} \in(0,1),(2) \text { and (3) imply } \\
& \qquad v_{R}^{1 R}=\frac{\theta_{R}-\theta_{1}+k}{k}, \quad V_{R}^{1 D}=\frac{\theta_{1}-\theta_{D}}{k}
\end{aligned}
$$

By the uniform distribution of voter preferences, $V_{R}=1-\tilde{\theta}_{j}$. Substituting $1-\tilde{\theta}_{j}$ for $v_{R}^{1 j}$ and $\tilde{\theta}_{j}$ for $\theta_{1}$ in $(10)$, we obtain that the unique cutpoints $\tilde{\theta}_{j}$ such that $V_{R}\left(\tilde{\theta}_{j}\right)=V_{R}^{1}$ for $\theta_{1}=\tilde{\theta}_{j}$, are given by:

$$
\tilde{\theta}_{R}=\frac{\theta_{R}}{1+k}, \quad \tilde{\theta}_{D}=\frac{\theta_{D}+k}{1+k}
$$

We use $\tilde{\theta}_{R}$ as a shorthand for the cutpoint strategy with $\tilde{\theta}_{R}$ as the cutpoint. Assume president $R$ is in of $\mathrm{fice}$.

It is direct to use single-peakedness (Lemma 1) to show that $\tilde{\theta}_{R}$ satisfies (C) for any $\left(S, \tilde{\theta}_{R}\right), S S I$, and that any $(I, z)$ that involves measurable deviations from $\tilde{\theta}_{R}$ falls to satisfy (C). By Proposition 1, then, $\left(I, \tilde{\theta}_{R}\right)$ belongs to the core and any $(I, z), z \neq \tilde{\theta}_{R}$, does not. ${ }^{10}$ Hence, by Greenberg's theorem, $\tilde{\theta}_{R}$ represents the unique SN.

The proof of (8) parallels that of (7), and (9) follows immediately from substitution of $\tilde{\theta}_{R}$ and $\tilde{\theta}_{D}$ into (2) and (3).

Q.E.D.

This result establishes that the policy outcome equals the ideal point of the voter at the legislative cutpoint. Every voter on the left of the 
cutpoint would want a policy more on the left, thus a reduction in $V_{\dot{R}}$ and vice versa.

This proposition also shows that voters use the legislative election to pull the incumbent president's policy away from the incumbent's most preferred policy. That is, in the second period the voters use the legislature to moderate the president. For this reason, a party receives more votes in the legislative elections when it does not hold the presidency than when it does. Note that Proposition 2 holds regardless of the positions of $\theta_{D}$ and $\theta_{R}$ relative to the median voter; the pivotal voter is always interior to the two parties, even if the two parties are botn located to the same side of the median.

If both parties are to the same side of the median, an immediate corollary to Proposition 2 is that the more extreme of the two parties can never win control of the legislature. On the other hand, if the two parties are on opposite sides of the median, the opposition can gain control of the legislature. "Divided government" becomes a possibility.

\section{3.d Presidential and legislative elections at $t=1$.}

For expositional purposes, let us consider first the case in which both the president and the legislature serve one period. This implies that we can consider the first period election in isolation. We first establish a useful preliminary result.

\section{Proposition 3}

Any pair $(I, z)$ is always dominated if $z$ leads to a tied presidential election.

\section{Proof: See Appendix}

The basic intuition of the proof is that the voters can more effectively 
moderate the executive if there is no uncertainty about the presidential election. Thus, a tied presidential race is always dominated by strategies which imply a sure winner with the "optimal" amount of legislative moderation. It follows that a tied presidential race cannot be $\mathrm{SN}$. Note that this result holds even with complete symmetry; that is, even with symmetric voter preferences, a symmetric distribution of voter ideal points, and party locations symmetric around the median voter. ${ }^{11}$

We can now show that if $S N$ equilibria exist they imply either $D$ winning the presidency with a legislative cutpoint $\tilde{\theta}_{D}$ and/or R winning the presidency and $\tilde{\theta}_{R}$.

\section{Proposition $\underline{4}$}

A necessary and sufficient condition for a pair (I,z) to be SN is either 1. The strategy $z$ implies $R$ is president with vote $M_{R z}>1 / 2$; the legislative component of $z$ is $\tilde{\theta}_{R}=\frac{\theta_{R}}{1+k}$; and (the following conditions insure that $\tilde{\theta}_{R}$ is not dominated by electing $D$ president):

1.A if $\theta_{D}+k \geq \tilde{\theta}_{R}$, the following condition is not satisfied:

$$
\text { [1.A1] } \tilde{\theta}_{\mathrm{R}}>\frac{1}{2} \text {. }
$$

1.B if $\theta_{D}+k<\tilde{\theta}_{R^{\prime}}$ there does not exist $x^{\prime}, \theta^{\prime}$ which satisfy

$$
\begin{aligned}
& \text { [1.Bi] } x^{*}=\theta_{D}+k v_{R}^{*} \\
& \text { [1.Bii] } v_{R}^{*}=1-\tilde{\theta}_{R}+\theta^{\prime} \\
& \text { [1.Biii] } u\left(x^{*}, \theta^{\prime}\right)=u\left(\tilde{\theta}_{R^{\prime}}, \theta^{\prime}\right) \\
& \text { [1.Biv] } \theta^{\prime}>\frac{1}{2} .
\end{aligned}
$$

2. The strategy $z$ implies $D$ is president with vote $M_{R z}<1 / 2$; the legislative component of $z$ is $\tilde{\theta}_{D}=\frac{\theta_{D}+k}{1+k}$. In addition, $z$ must satisfy conditions symmetric to those given for $R$ president. 


\section{Proof: See Appendix.}

\section{Corollary}

There is a unique SN outcome when elther $\tilde{\theta}_{R} \leq 1 / 2$ or $\tilde{\theta}_{D} \geq 1 / 2$.

The corollary shows that we have a unique SN when both legislative cutpoints are to the same side of the median, with the cutpoint closer to the median corresponding to the stable outcome. Of course, both cutpoints are to the same side of the median whenever the two party bliss points are to the same side of the median.

\section{Remarks}

1. In the case of symmetric preferences, condition [1.Biii] becomes $\theta$, $=\left(x^{*}+\tilde{\theta}_{R}\right) / 2 \equiv \mathrm{r}$. Analogously, for domination of $\tilde{\theta}_{D}$ we would have $\theta^{\prime}=$ $\left(\mathrm{x}^{*}+\tilde{\theta}_{\mathrm{D}}\right) / 2 \equiv \mathrm{d}$. After simple algebra, we obtain:

$$
\begin{gathered}
r=\frac{\theta_{R}(1-k)+\left(\theta_{D}+k\right)(1+k)}{(2-k)(1+k)} \\
d=\frac{\left(\theta_{R}-k\right)(1+k)+\left(\theta_{D}+k\right)(1-k)}{(2-k)(1+k)}
\end{gathered}
$$

The relationships $r=1 / 2, \quad r<\tilde{\theta}_{R}$ and $d=1 / 2, d>\tilde{\theta}_{D}$ then define the parameter regions where a SN exists. These are shown in Figure $1 B$ for $k=0.2$. It can be seen that for small $k$ and the parties reasonably symmetric about the median but far from the median, both $R$ winning the presidency and $D$ winning the presidency are SN. The "objecting" party is too far from the median, relative to the amount of moderation possible, to upset the presidential victory of the other party. For larger values of $k$ (such as 0.4), this situation cannot occur, as illustrated by Figure 1C. In fact, for all $k>0$, with the parties close to full symmetry around the median and sufficiently close to the median, a SN does not exist. 
2. Examination of the conditions in Proposition 4 thus shows that the Greenberg core to this voting game can be empty, be characterized by one outcome, or be characterized by two outcomes. Note, however, that condftions [1.B] for domination of an equilibrium with an $R$ president imply that voters with ideal points below the policy $\left(x^{*}\right)$ to be implemented if $D$ becomes president are required to vote $R$ for Congress as domination occurs. Such voters are not behaving consistently with condition (C) of Proposition 1: consistent behavior impliesthat they should vote DD. (See Figure 2.) Thus, the absence of a core for some parameter values is not troublesome if we can show that elimination of this kind of inconsistency always reestablishes either $\tilde{\theta}_{R}$ or $\tilde{\theta}_{D}$ as a policy outcome in the VN\&M abstract stable set. This is precisely the implication of the following proposition.

\section{Proposition $\underline{5}$}

An abstract stable set always exists and contains at least one pair $(I, z)$. The pair $(S)(I, z)$ which are in the stable set must have party $R$ winning the presidency with certainty and $\tilde{\theta}_{R}$ as the cutpoint of the legislative elections and/or party D winning the presidency with certainty and $\tilde{\theta}_{D}$ as the cutpoint of the legislative elections. ${ }^{12}$

Proof: See Appendix.

\section{Remarks}

1. When $(I, z)$ contains a cutpoint presidential rule $\hat{\theta}<1 / 2$, and $\tilde{\theta}_{R} \leq$ $1 / 2,(1, z)$ is in $K$ and $\tilde{\theta}_{D}$ is not in $K$. Similarly, $\hat{\theta}>1 / 2, \tilde{\theta}_{D} \geq 1 / 2$ is $\dot{i}_{11} K$ and $\tilde{\theta}_{R}$ is not in $K$.

2. A pair ( $I, z)$ implytng a tie in the presidential election is not in the stable set. The only pair $(s)(I, z)$ included in the set imply a certain 
presidential election and a policy outcome of $\tilde{\theta}_{R}$ or $\tilde{\theta}_{D}$.

3. In the case of symmetric preferences, the analogs to (12) and (13) are:

$$
\begin{aligned}
& r^{\prime}=\frac{\theta_{R}+\left(\theta_{D}+k\right)(1+k)}{(2+k)(1+k)} \\
& d^{\prime}=\frac{\left(\theta_{D}+k\right)+\theta_{R}(1+k)}{(2+k)(1+k)}
\end{aligned}
$$

The equations $r^{\prime}=1 / 2$ and $d^{\prime}=1 / 2$ define, respectively, the regions where $R$ winning the presidency with outcome $\tilde{\theta}_{R}$ and $D$ winning with $\tilde{\theta}_{D}$ are supported by members of the abstract stable set. Figures $1 D$ and $1 E$ illustrate that at least one of the two outcomes is always supported. When the two parties are reasonably symmetric about the median, both are supported.

Thus, in summary:

If $\tilde{\theta}_{D}<\tilde{\theta}_{R} \leq \frac{1}{2}$ party $R$ wins the presidency with certainty, and the equilibrium outcome is unique.

If $\tilde{\theta}_{\mathrm{R}}>\tilde{\theta}_{\mathrm{D}} \geq \frac{1}{2}$ party $\mathrm{D}$ wins the presidency, and the equilibrium outcome is unique.

(iii) If $\tilde{\theta}_{D}<\frac{1}{2}<\tilde{\theta}_{R}$ there are two possible equilibria. In one $R$ wins the presidency with $\tilde{\theta}_{R}$ as in ( 1 ) and in the other $D$ wins the presidency and $\tilde{\theta}_{D}$ is as in (ii), but, depending on parameters, only one of these may be vN\&M.

(iv) If the parties locate symmetrically about the median $\left(\theta_{R}+\theta_{D}=1\right)$ and preferences are symmetric, $\tilde{\theta}_{R}$ and $\tilde{\theta}_{D}$ are both equilibria.

In cases ( $i i i)$ and (iv), there is always split ticket voting. We have RD split tickets for $\hat{\theta}>\frac{1}{2}$ and DR split tickets for $\hat{\theta}<\frac{1}{2}$. That is, the 
party of the winning president recelves fewer votes in the legislative elections than in the presidential election.

In the less realistic cases ( 1 ) and (1i), $\hat{\theta}$ may be on either side of the legislative cutpoint. Split ticket voting will occur except in the kntfe-edge case of $\hat{\theta}=\tilde{\theta}$.

2.3e President and legislature elected simultaneously in period $1 ;$ the President serves two periods with an additional legislative election in period $\underline{2}$

In the two period game, a voter's cholce in the presidential election depends upon the expected policies at $t=2$. We assume that in period one the voters rationally expect the policies corresponding to the SN outcomes for $t=2$. This is equivalent to the following:

A1 at $t=1$, the second period strategy components are restricted to the SN strategy in the $t=2$ subgame.

In our setup with no uncertainty, using $\mathbf{A}$ it is immediate to establish that the the equilibrium cutpoint of legislative elections in period 1 are identical to those of period 2 . Since information is complete, the result of the first period elections does not provide any additional information which could change voters' behavior in the second period. Thus, the abstract stable set characterized in Proposition 5 also applies to the two period game.

\section{Proposition 6}

With complete information, the legislative vote is identical in both periods; there is no mid-term congressional cycle. 


\section{GENERALIZATIONS OF THE COMPLETE INFORMATION MODEL}

This section shows that our results survive, at least qualitatively, if we relax several simplyfying assumptions adopted thus far.

i) Impact of the legislature on policy.

We can relax the assumption of linearity and postulate that policies are given by:

$$
\begin{gathered}
x^{D}=\theta_{D}+g_{D}\left(V_{R}\right) \\
x^{R}=\theta_{R}-g_{R}\left(1-V_{R}\right)
\end{gathered}
$$

where $g_{R}(\cdot)$ and $g_{D}(\cdot)$ are strictly increasing in their arguments, continuous and twice differentiable, and $g_{R}(0)=g_{D}(0)=0$. In keeping with (4), we also assume that for every value of $V_{R}$ the following holds:

$$
\theta_{D}+g_{D}\left(V_{R}\right)<\theta_{R}-g_{R}\left(1-V_{R}\right)
$$

\section{ii) Distribution of voter ideal points.}

We need only the assumption that this distribution is continuous and twice differentiable on $[0,1]$. Consequently, if $\theta$ is a cutpoint, we can write,

$$
\mathrm{V}_{\mathrm{R}}=1-\mathrm{H}(\theta)
$$

where $\mathrm{H}$ is strictly decreasing and twice differentiable.

Even under these general assumptions, one important feature of Proposition 1 remains unaffected: preferences are single-peaked in $V_{R}$ and a necessary condition for SN equilibria in the legislative elections at $t=2$ is that the equilibrium policy equals the ideal point of the pivotal voter. For instance, suppose $D$ holds the presidency. Then, $\tilde{\theta}_{D}$ satisfies the following: ${ }^{13}$

$$
x^{D}=\tilde{\theta}_{D}=\theta_{D}+g_{D}\left(1-H\left(\tilde{\theta}_{D}\right)\right)
$$

An analogous result holds when $R$ is in office:

$$
x^{R}=\tilde{\theta}_{R}=\theta_{R}-g_{R}\left(H\left(\tilde{\theta}_{R}\right)\right)
$$


Since $g_{D}(\cdot)$ and $g_{R}(\cdot)$ are strictly monotone, the implicit functions (20) and (21) imply unique solutions for $x^{D}$ and $x^{B}$. Note that the proof of Proposition 1 relies only on the single-peakedness of voters' preferences on $V_{R}$, which is malntalned here. It is also imnediate to establish the following inequalities:

$$
\theta_{D}<\tilde{\theta}_{D}<\tilde{\theta}_{B}<\theta_{B}
$$

The first and third inequalities follow immediately from the assumption of continulty of the distribution voters' Ideal points, which implies that there will always exist some voters who want to "moderate" the president. The second Inequality follows from (18) and from the fact that $V_{R}^{D}>V_{R}^{R}$, where $V_{R}^{j}$ indicates the legislative vote for party $R$ when president $j$ is in office. ${ }^{14}$

Condition (22) also implles that the results of sections $2.3 \mathrm{c}, \mathrm{d}$ and $e$ also apply (qualitatively) in the general case. As before, when presidential and legislative elections are held simultaneously, there are only two possible equllibria, both with ticket splitting voters. In one equilibrium $R$ wins the presidency and $\tilde{\theta}_{F}$ is the cutpoint of the legislative election. In the other $D$ wins the presidency and $\tilde{\theta}_{D}$ is the cutpoint for the legislature, which, as before, is used to moderate the president. The argument used previously to rule out tied presidential elections continues to hold.

Finally the conditions under whlch the two possible equilibrla are SN or VN\&M are in general different from those derived in Propositions 4 and 5 . However the basic features of these conditions should be unchanged: for instance, the more unbalanced toward the left are the bliss points of the candidates relative to the median voter, the more likely it is that $R$ wins the presidency and $\tilde{\theta}_{B}$ is the equilibrium cutpoint of the legislative elections. This similarity with the case examined in Section 2 is 
particularly evident if $g_{D}(\cdot)=g_{R}(\cdot)$. If, instead, $g_{D}(\cdot) \neq g_{R}(\cdot)$ there is an additional asymmetry in the system, due to the different degree in which the two presidents can be moderated. This asymmetry, together with the degree of asymmetry in the location of $\theta_{R}$ and $\theta_{D}$ with respect to the median affects whether $\tilde{\theta}_{\mathbf{R}}$ or $\tilde{\theta}_{D}$ represent stable outcomes. In fact, if, say, president $R$ is more influenced by the legislature than $D$, then, ceteris paribus, it is most likely that the equilibrium with R president and $\tilde{\theta}_{R}$ the legislative cutpoint is VN\&M stable. This is because the voters could more easily achieve "moderate" outcomes by electing the president who is more easily influenced by the legislature. In general, it may be difficult or impossible to derive these conditions analytically. As in Section 2 , a mid-term cycle in congressional votes is ruled out by the assumption of complete information.

\section{THE MODEL WITH UNCERTAINTY}

This section considers an example in which the distribution of voters' preferences is perturbed by a random shock. We show the existence of an equilibrium with a mid-term cycle. To construct this example, we return to the simplest case, with all the simplifying assumptions and notation of Section 2. We also assume quadratic preferences:

$$
u_{t j}=-(1 / 2)\left(x_{t}-\theta_{j}\right)^{2} \quad j=D, R, i
$$

The extremes of the uniform distribution of voters ideal points are now given by $I_{a}=[a, 1+a]$ where $a$ is a random varlable with zero mean distributed uniformly on the interval [-w, w] with:

$$
W<\theta_{D}<\theta_{R}<1-W
$$

Thus, for any realization of a there are voters with bliss points on 
both sides of $\theta_{R}$ and $\theta_{D}$. The distribution of a is "common knowledge". Independent draws of a $\underline{\text { are made }} \underline{\underline{t}} \underline{t} \equiv \underline{1}$ and $\underline{t} \equiv \underline{2}$. The set of possible voter types is contained in the interval $I_{w}=[-w, 1+w]$. In this section, equilibrium strategies are specified in terms of $I_{n}$. Finally we assume that voters cannot communicate thelr preferences to each other.

Consider first the legislative elections at $t=2$. The analysis parallels Section 2.3.c. The Republican vote for the legislature, which now depends on the realization of $a$, is denoted $V_{R}(a)$. The expected utility of voter 1 , with a bliss point $\theta_{1}$ such that $\theta_{R} \leq \theta_{1} \leq \theta_{D}$ when a president $R$ is in office in perlod 2 is given by:

$$
E u_{1}^{R}=\int_{\underline{a}}^{\bar{a}}-\frac{1}{2}\left(\theta_{R}-k\left(1-V_{R}(a)\right)-\theta_{1}\right)^{2} \frac{1}{2 w} d a
$$

An analogous expression for $E u_{1}^{D}$ holds if president $D$ is in office. Assumption (24) is key to (25). Voters with bliss points $\theta_{1}$ such that $\theta_{1}\left\langle w\right.$ or $\left.\theta_{1}\right\rangle$ $1-w$, by observing their preferences and knowing that the random variable a is distributed uniformly, would update, and, as a result, their expected value of a would not be zero. Condition (24) implies that these voters have dominant strategies for every realization of a: they always vote RR or DD respectively. Thus, their updating does not affect the equilibrium analysis.

Denote with $\tilde{\theta}_{j 2}$ the legislative cutpoint in period 2 when party $j$ holds the presidency. Also let $E V_{R}^{1 /}$ denote i's indirect bliss point for R's expected vote at $t=2$ when party $J$ has won the presidency at $t=1$. The $t=2$ results for the second period subgame are given in the following proposition. Proposition 7

A necessary and sufficlent condition for $(S, z)$ to be undominated in the $t=2$ sub-game is: 
$\left(C^{2}\right)$

for all measurable TCS such that $i \in T$ votes $R, E V_{R}^{j}(z) \leq E V_{R}^{1 j}$

for all measurable LeS such that $i \in L$ votes $D, \operatorname{EV}_{R}^{j}(z) \geq{E V_{R}^{1 j}}^{j}$.

Moreover,

$\tilde{\theta}_{R 2}^{*}=\frac{\theta_{R}}{1+K}$ is the unique SN equilibrium when a president $R$ is in office

$\tilde{\theta}_{D 2}^{*}=\frac{\theta_{D}^{+k}}{1+k}$ is the unique SN equilibrium when a president $D$ is in office

$\mathrm{x}^{\mathrm{R} 2}=\frac{\theta_{\mathrm{R}}}{1+\mathrm{k}}+\mathrm{ka}$ is the policy with a $\mathrm{R}$ president

$\mathrm{x}^{\mathrm{D} 2}=\frac{\theta_{\mathrm{D}}+\mathrm{k}}{1+\mathrm{k}}+\mathrm{ka}$ is the policy with a $\mathrm{D}$ president

$\tilde{\theta}_{\mathrm{R} 2}^{*}=E\left(x^{\mathrm{R} 2}\right) ; \quad \tilde{\theta}_{\mathrm{D} 2}^{*}=E\left(x^{\mathrm{D} 2}\right)$

Proof: See Appendix.

The analogy with the complete information case is that the expected policy is equal to the ideal policy of the pivotal voter $\left(\tilde{\theta}_{\mathrm{R} 2}^{*}\right.$ or $\left.\tilde{\theta}_{D 2}^{*}\right)$.

Let us now turn to the first period election. It is convenient to express $P$, the probability of an $R$ victory in the presidential context, as $P(\hat{\theta})$, where $\hat{\theta}$ is the presidential cutpoint. We will construct an equilibrium such that $P \in(0,1)$.

Let $E V_{R}^{i P(\hat{\theta})}$ denote $I^{\prime} s$ expected vote bliss point at $t=1$ given $\hat{\theta}$. Proposition (7) can be directly extended to show that:

Lemina 2

A necessary and sufficient condition for $(S, z)$ to be undominated for a given and fixed $\hat{\theta}$ is 
for all measurable $T C S$ such that $1 \in T$ votes $R$ in the $t=1$ legislative $\left(C^{1}\right)$ elections, $E_{R}(z) \leq E V_{R}{ }^{1 P(\hat{\theta})}$ and

for all measurable LCS such that $i \in L$ votes $D$ in the $t=1$ legislative elections, $\operatorname{EV}_{R}(z) \geq \operatorname{EV}_{R}^{1 P(\hat{\theta})}$

Using Lemma 2 and the results developed in the certainty model, the following result is immediate:

Lemma $\underline{3}$

For $(I, z)$, given fixed $\hat{\theta}$, the only undominated cutpoint at $t=1, \tilde{\theta}$, is given by:

$$
\tilde{\theta}=P(\hat{\theta}) \tilde{\theta}_{R}+(1-P(\hat{\theta})) \tilde{\theta}_{D}
$$

Condition (26) and the uniform distribution of a imply:

$$
\tilde{\theta}(\hat{\theta})= \begin{cases}\tilde{\theta}_{R} & \hat{\theta} \leq \frac{1}{2}-w \\ \frac{\left(\frac{1}{2}+w-\hat{\theta}\right)}{2 W}\left(\theta_{R}-k-\theta_{D}\right)+\theta_{D}+k & \frac{1}{2}-w<\hat{\theta}<\frac{1}{2}+w \\ \tilde{\theta}_{D}(1+k) & \frac{1}{2}+w \leq \hat{\theta}\end{cases}
$$

The next result follows immediately from (27).

\section{Proposition $\underline{8}$}

Given $0<P(\hat{\theta})<1$, a necessary rondition for non-domination for $\hat{\theta} \mathbf{f}$ ixed is that the unique legislative cutpoint $\tilde{\theta}$ satisfles the following:

$$
\tilde{\theta}_{\mathrm{R} 2}<\tilde{\theta}<\tilde{\theta}_{\mathrm{D} 2}
$$

That is, satisfying $\left(C^{1}\right)$ with uncertain presidential elections implies a 
mid-term congressional cycle: the party of the president always has lower expected plurality in mid-term legislative elections, than in the legislative elections held at the same time as the presidential elections.

The intuition of this result is straightforward. If say, $\tilde{\theta}<\tilde{\theta}_{D 2}$ then voters with bliss points $\theta_{1}$ such that $\tilde{\theta}<\theta_{1}<\tilde{\theta}_{D 2}$ (and $\theta_{i}>\theta_{D}$ ) act as follows: in the first period under uncertainty about the president's identity, these voters vote $R$ for the legislature. This implies that they would want to "moderate" a D president despite the fact that they are taking. the risk of making a $R$ president less constrained, if $R$ wins. Then, in the second period, when they know that a $D$ president is in office, they switch to voting $D$ for Congress, reducing the moderation of the $D$ president. This behavior cannot be rational, thus $\tilde{\theta}<\tilde{\theta}_{\mathrm{D} 2}$ cannot be an equilibrium. Analogous argument holds for $\tilde{\theta}>\tilde{\theta}_{R 2}$. Finally note, from (27), that if $P(\hat{\theta})=$ 1 (or $P(\hat{\theta})=0$ ), we have $\tilde{\theta}=\tilde{\theta}_{R 2}$ (or $\tilde{\theta}=\tilde{\theta}_{D 2}$ ). Thus, with no uncertainty about the presidential outcome, the legislative cutpoints are the same in both periods; as in the complete information model we do not have a midterm cycle.

Let us now turn to the presidential vote. A necessary condition for $(S, z)$ undominated when $O<P(\cdot)<1$ is obviously

for all measurable $T \in S$ such that $i \in T$ votes $R$ in the presidential election, $E U_{i}(R$ president|legislative components of $z$ )

$\left(C^{P}\right)$ $\geq E U_{1}$ (D president/legislative components of $z$ )

for all measurable LeS su, $\zeta$ that $l \in L$ votes $D$ in the presidential election, $E U_{1}$ ( $R$ president|legislative components of $z$ ) $\leq E U_{1}$ (D president|legislative components of $z$ )

Equating the two expected utilities in $\left(C^{P}\right)$, and using $\mathbf{A}$, we find the presidential election cutpoint $\hat{\theta}$ by solving the following: 


$$
\begin{gathered}
E\left[-\left(\theta_{R}-k \tilde{\theta}+k a-\hat{\theta}\right)^{2}-\beta\left(\frac{\theta_{R}}{1+k}+k a-\hat{\theta}\right)^{2}\right] \\
=E\left[-\left(\theta_{D}+k(1-\tilde{\theta})+k a-\hat{\theta}\right)^{2}-\beta\left(\frac{\theta_{D}+k}{1+k}+k a-\hat{\theta}\right)^{2}\right]
\end{gathered}
$$

The unique solution of (29) is:

$$
\hat{\theta}(\tilde{\theta})=\frac{\left(\theta_{D}+\theta_{R}+k\right)\left(\beta+(1+k)^{2}\right)-2 k(1+k)^{2} \tilde{\theta}}{2(1+k)(1+k+\beta)} \quad \theta_{D}<\tilde{\theta}<\theta_{R}
$$

Note that $\theta_{D}<\hat{\theta}<\theta_{R}$.

We now develop an important condition on the presidential strategy components given the legislative vote. Let $P(z)$ denote the probability that $R$ is president for strategies $z$.

\section{Lemma $\underline{4}$}

Given that the legislative components of strategies are fixed at $\tilde{\theta}, \tilde{\theta}_{D 2}^{*}$ $\tilde{\theta}_{\text {R2' }}^{*}$ a necessary and sufficient condition for $(S, z)$ to be undominated by $(T, y)$ when $O<P(z)<1$ and $0<P(y)<1$ is that the presidential component of $z$ is represented by the cutpoint $\hat{\theta}$ given by (30).

Proof: Except for the voter type $\theta_{1}=\hat{\theta}$, all voters in the presidential election have strictly dominant strategies given $\tilde{\theta}, \tilde{\theta}_{R 2}^{*}$ and $\tilde{\theta}_{D 2}^{*}$ ift $0<P(\cdot)<$ 1.

Q.E.D.

Since (30) is monotone on $\left(\theta_{D}, \theta_{R}\right)$ and $\tilde{\theta}(\hat{\theta}) \in\left(\tilde{\theta}_{D}, \tilde{\theta}_{R}\right)$ and is monotone, it is immediate to show that (30) and (27) have a solution. There is a unique, interior solution if:

A2. $\hat{\theta}\left(\tilde{\theta}_{R}\right)>\frac{1}{2}-w$ and $\hat{\theta}\left(\tilde{\theta}_{D}\right)<\frac{1}{2}+w$. 
Using (27) and (30), one can readly write the assumption explicitly in terms of the parameters of the model. In fact, by doing so it can be checked that the range of parameter values for which $\mathbf{A 2}$ is satisfied is quite large.

We are now ready to establish the following result.

\section{Proposition 9}

If $\mathbf{A 2}$ holds, an abstract stable set exists. This set contains a unique pair of the form $\left(I_{w}, z\right)$. This pair is denoted $\left(I_{w}, z^{*}\right)$ and corresponds to the cutpoints $\hat{\theta}^{*}$ and $\tilde{\theta}^{*}$, given by the unique solution of (27) and (30), and $\tilde{\theta}_{R 2}^{*}$ and $\tilde{\theta}_{D 2}^{*}$, given in Proposition 7.

Proof: See Appendix.

\section{Remarks}

1. Even when $\mathbf{A 2}$ is satisfled, for some parameter values, there is no $\mathrm{SN}$ in the presence of incomplete information. This can be demonstrated by assigning fixed numerical values to the parameters of the model, solving (27) and (30) for the two $t=1$ "* cutpoints and then finding, using computer grid search, alternative "'" cutpoints such that voter types at the "' cutpoints have an increase in expected utility. Since all voters have quadratic utility, it follows that all voters in the interval spanned by the "," cutpoints have higher utility than at the "cutpoints. Hence ( $\left.i, z^{*}\right)$ is dominated. On the basis of our computer analysis, we conjecture that, for every set of parameters satisfying $\mathbf{A 2}$, there is no SN.

2. If and only if $\theta_{R}+\theta_{D}=1$ there is no split ticket voting in the first period election. In this case only, simple algebra on (27) and (30) establishes that:

$$
\hat{\theta}^{*}=\tilde{\theta}^{*}=\frac{1}{2}
$$


3. If $\hat{\theta}^{*}>\frac{1}{2}$, that is $P\left(\hat{\theta}^{*}\right)<\frac{1}{2}$, the equilibrium implies three types of voters: $R R, D R, D D$. If $\hat{\theta}<\frac{1}{2}$ the three types are RR, RD, DD. Thus the voters who split their ticket are always a fraction of those voting for the presidential candidate more likely to win. In addition, the less uncertain is the presidential election (1.e. the larger $\left|\hat{\theta}^{*}-\frac{1}{2}\right|$ ) the larger the fraction of split tickets.

4. An interior solution with $0<P(\cdot)<1$ occurs only if there is "enough uncertainty" in the sense that $\mathbf{A} 2$ is satisfled. Parameter values for which this occurs are shown in Figure $3 \mathrm{~A}$. Figures $3 \mathrm{~B}$ and $3 \mathrm{C}$ illustrate cases in which $A 2$ is violated. Figure $3 B$ shows an unstable interior solution to (27) and (30). In this case voters can achieve an equilibrium outcome with no uncertainty in the presidential election. The heavy black lines show an infinity of cutpoint outcomes that correspond to the two VN\&M ( $\tilde{\theta}_{R}$ and $\left.\tilde{\theta}_{D}\right)$ of the game of complete information. The interior intersection is unstable in the sense that the cutpoints defined by the solution to (27) and (30) are "credibly" dominated. Finally, Figure $3 C$ illustrates the absence of an interior solution. The heavy black line now indicates a vN\&M where the party closest to the median wins the presidential election with certainty.

5. A necessary condition for $\mathbf{A} 2$ is given by the relative slopes of (27) and (30). This condition is: $W>\frac{\left(\theta_{R}-\theta_{D}-k\right) k}{2(1+k+\beta)}$. For $\theta_{R}$, $\theta_{D}$ fixed, the largest. $W$ needed to satisfy this inequality occurs for some value of $k$ intermediate between 0 and 1 . As $k \rightarrow 0$, executive influence on policy becomes predominant. Since there is little scope for moderation, there is little incentive to engage in strategic voting. As $k \rightarrow \theta_{R}-\theta_{D}$, its maximum value, the legislative influence on pollcy becomes predominant. The legislative vote can be used to achieve almost any desired expected policy outcome. The 
expected outcome will not be greatly influenced by the presidential outcome. Again there is little incentive to eliminate the uncertainty in the presidential election.

6. The case in which both the president and legislature are elected every period is obtained by setting $\beta=0$ in $(30)$. The cutpoints ( $\hat{\theta}$ and $\tilde{\theta}$ ) in this case describe the electoral equilibrium in every period. Proposition 9 applies to this case as well. Note that in this case assumption $A 1$ is irrelevant.

\section{CONCLUSIONS}

This paper extends the spatial theory of voting to an institutional structure in which policy choices are a function of the composition of the legislature and of the executive. A game between the voters is considered; in fact, in an institutional setup in which the policy outcome depends upon relative plurality, each voter has incentives to be strategic since the outcome depends upon how everybody else votes.

The model has several testable implications which appear to be consistent with some observed patterns of voting behavior in the United States and perhaps in other democracies in which the executive is directly elected. For instance, the model predicts: a) split-ticket voting; b) for some parameter values, a split government with different parties controlling the executive and holding a majority in the legislature; and c) the mid-term electoral cycle. The split government prediction would also apply to nations like France where the executive and legislative are not elected simultaneously. One caveat is the very pervasity of the mid-term cycle in the United States which occurred even in those cases where there was little 
uncertainty about who would win the presidency. Thus, while this model appears to be far richer than the standard two party competitive model, future work would hopefully lead to a more complete model.

In fact, several extensions of the basic model should be possible. First, abstentions should be considered as an additional moderating device, for instance if supporters of the winning presidential candidate do not vote in legislative elections.

Second, one could consider a party's platform as a strategic variable, rather than an exogenously given ideal point. These platforms become strategic variables if parties can make binding precommittments to their preelectoral platform or if these precommittments can be achieved if the two parties select candidates in primaries with bliss polnts corresponding to the desired platforms. If the party can credibly choose policies before the election, and there is uncertainty about voters' preferences, in a model with no legislature, the two candidates choose, in general, non fully convergent platforms. [See Wittman (1977, 1988) and Calvert (1985)]Thus, there would always be voters with ideal points in between the two platforms and these voters would want to "moderate", as in our model. Needless to say, in choosing their platform the presidential candidates would take account of these moderating strategies. In fact, the expected influence of the legislature may create an incentive for the two partles to diverge even more, if they know that whichever party holds the presidency must compromise with the legislature.

Third, this model may have implications for the issue of entry of thire parties [Palfrey (1984), Greenberg and Shepsle (1987)]. Consider the case in which the 1deal points of the two parties are on opposite sides of the 
median. The "threat" of entry of a third party in the middle pursuing "moderate" policies may be reduced if the voters can achieve some degree of moderation of policy in a two party system.

Fourth, the basic idea of strategic moderation could also be applied to parliamentary systems, where the executive is not directly elected. ${ }^{15}$ Strategic voting would be even more essential in multi-party parliamentary system where the coalitions of parties which can be formed in the legislature depend upon the distribution of seats or of vote shares. 


\section{ENDNOTES}

${ }^{1}$ For surveys of this literature see Enelow and Hinich (1984) and Ordeshook (1986).

${ }^{2}$ The existence of "polarization" in American politics has been widely documented by Poole and Rosenthal (1984a,b; 1985).

${ }^{3}$ This phenomenon has occurred repeatedly in the United States and recently in France with the "cohabitation" of a Socialist President and a conservative legislature.

${ }^{4}$ Erikson (1988) tests coattails and other alternative explanations of the mid-term cycle and finds that they are not supported by the data.

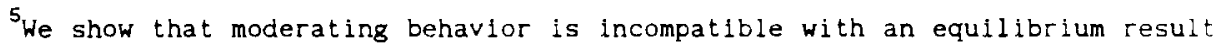
of a tied Presidential election.

${ }^{6} \mathrm{~A}$ more general interpretation of the parameter $\mathrm{k}$ would be given by writing $\mathrm{k}$ as a function of $\theta_{R}$ and $\theta_{D}$ as follows:

$$
k=\psi\left(\theta_{R}, \theta_{D}\right)\left(\theta_{R}-\theta_{D}\right)
$$

with $0<\psi(\cdot)<1$ for $0 \leq \theta_{R} \leq 1$ and $0 \leq \theta_{D} \leq 1$. This specification clarifies the effect of the legislature on policy outcomes as a function of the ideal policies of the two parties. Since $\theta_{R}$ and $\theta_{D}$ are taken as exogenous in this paper, the results would be unchanged if we used $F$. I for $k$. A much more insightful and important role would be played by the functional dependency of $k$ on $\theta_{R}$ and $\theta_{D}$ in a model where party position we: endogenously chosen as in Wittman (1988) and Calvert (1985). More on this point in the conclusions. 
${ }^{7}$ This institutional structure approximates not only the United States system, but, generally, all systems (e.g. France) with direct Presidential and legislative elections; in fact, our model can handle both the cases in which the elections are simultaneous and the case in which they are staggered. Needless to say, we abstract from many other important institutional aspects, in particular the district system, and the fact that Congress is not completely reelected every two years.

${ }^{8}$ We need only consider measurable subsets since no non-measurable subset can affect outcomes and thus individual utilities.

${ }^{9}$ It should be emphasized that elimination of weakly dominated strategies is not sufficient to select cutpoint outcomes. In fact even though voters with bliss points $\theta_{1} \leq \theta_{D}$ and $\theta_{1} \geq \theta_{R}$ have weakly dominant strategies (DD and $R R$ respectively) the other voters do not have weakly dominant strategies in presidential elections.

${ }^{10}$ Since the SN are a subset of the VN\&M equilibria, (I, $\tilde{\theta}_{R}$ ) is also VN\&M. Moreover, there is no other abstract stable set with some $(I, z) z \neq \tilde{\theta}_{R}$, since $z$ would not satisfy both internal and external stability.

${ }^{11}$ Note that in this situation sincere voting, as in Fiorina (1988), would lead to 50-50 splits in both the presidential and legislative vote. 
12 While we have not proved the abstract stable set is unique, Greenberg (1989) shows that $K$ is unique for a finite number of players. With a finite number of players, we can also construct a $K$ with properties virtually identical (except for integer problems) to those found for the continuum. Thus, for a large but finite number of players, there would be a unique $K$ with qualitatative properties identical to those developed for the continuum.

${ }^{13}$ Consider the utility function of voter $i$. Setting the first derivative of his utility respect to policy equal to zero implies: $\theta_{1}=\theta_{D}+g_{D}\left(1-H\left(\tilde{\theta}_{D}\right)\right)$. Imposing the equilibrium condition $\theta_{i}=\tilde{\theta}_{0}$ leads to (20).

${ }^{14}$ Suppose instead, $V_{R}^{R}>V_{R}^{D}$. This implies that some voters vote $R$ for the legislature when party $R$ controls the presidency and $D$ when $D$ is president. This strategy is inconsistent with single-peaked individual preferences.

${ }^{15}$ Austen-Smith and Banks (1988) have investigated a three party parliamentary system with a strategic voting model. The process that links voting to policy in their model follows an approach that is quite different from ours. 


\section{REFERENCES}

Alesina, Alberto (1988). "Credibłl1ty and Pollcy Convergence in a Two-Party System with Rational Voters", Amerlcan Economic Review, 78, (4), 796-806.

Alesina, Alberto and Howard Rosenthal (1989). "Partisan Cycles in Congressional Elections and the Macroeconomy", American Political Sclence Review, Vol. 83 (June) 373-98.

Aumann, Robert (1959). "Acceptable Polnts in General Cooperative n-Person Games" in Contributions to the Theory of Games. Princeton University Press: Princeton, NJ.

Austen-Smith, David and Jeffrey Banks (1988). "Elections, Coalitions, and Legislative Outcomes", Amerlcan Political Science Review, Vol. 82 (June) 407-422.

Bernheim, Douglas, Bezael Peleg and Michael Whinston (1987). "Coalition Proof Nash Equilibria. 1. Concepts", Journal of Economic Theory, Vol. 2. 1-12 (June).

Calvert, Randal1 (1985). "Robustness of the Multidimensional Voting Model, Candidate Motivations, Uncertainty and Convergence", American journal of Political Science, Vol. 29, 69-95, (February).

Enelow, James and Melvin Hinlch (1984). Spatial Analysis of Elections, Cambridge University Press: New York.

Erikson, Robert (1988). "The Puzzle of Midterm Loss", Journal of Politics, Vol. 50, 1012-1029.

Fiorina, Morris (1988). "The Reagan Years: Turning to the Right or Groping Toward the Middle", in B. Cooper et al. eds., The Resurgence of Conservatism in Anglo-Amertcan Democracies, Duke University Press: Durham, NC.

Greenberg, Joseph (1989). "Deriving Strong and Coalition Proof Nash Equilibrla from an Abstract System", Journal of Economic Theory, forthcoming.

Greenberg, Joseph and Kenneth Shepsle (1987). "The Effect of Electoral Rewards in Multiparty Competition with Entry". American Political Sclence Review, Vol. 81, 525-37 (June).

Hammond, Thomas and Gary Miller (1988). "The Core of the Constitution", American Political Science Review. Vol. 84, 1155-1174 (December).

Ordeshook, Peter (1986). Game Theory and Political Theory, Cambridge University Press: Cambridge, UK. 
Palfrey, Thomas R. (1984). "Spatial Equllibrium with Entry", Review of Economic Studies, Vol. 51, 139-56.

Poole, Keith T. and Howard Rosenthal (1984a). "U.S. Presidential Elections 1968-1980: A Spatlal Analysis", American Journal of Political Science. Vol. 28, 283-312, (May).

Poole, Keith T. and Howard Rosenthal (1984b). "The Polarization of American Politics", Journal of Politics, Vol. 46, 1061-1079.

Poole, Keith T. and Howard Rosenthal (1985a). "A Spatial Model for Legislative Roll Call Analysis", American Journal of Political Science, Vol. 29, 357-84.

Shepsle, Kenneth (1986). "The Positive Theory of Legislative Institutions: An Enrichment of Soclal Choice and Spatial Models". Publis Choice, 50 , 135-78.

Wittman, Donald (1977). "Candidates with Policy Preferences: A Dynamic Model", Journal of Economic Theory, Vol. 14, 180-189, (February).

Wittman, Donald (1988). "Spatlal Strategies When Candidates Have Policy Preferences" in Enelow, Janes and Melvin Hinich, eds., Readings in the Spatial Theory of Elections, Cambridge University Press, forthcoming. 


\section{APPENDIX}

\section{Proof of Proposition $\underline{3}$}

A tie generates a lottery between two outcomes $x^{R}$ and $x^{D}$. Three cases are possible:

1. $u\left(x^{R}, 1 / 2\right)>u\left(x^{D}, 1 / 2\right)$; recall that $\theta_{1}=1 / 2$ for the median voter.

Since all voters have identical preferences except for the location of the bliss point, it must be the case that there exists $\xi<1 / 2$ such that $u\left(x^{R}, \theta_{1}\right)>u\left(x^{D}, \theta^{1}\right)$ for all $\theta_{1} \geq \xi$. Since the presidential vote is tied under $z$, some of these voters must have voted $D$ for president under $z$. If the $D$ voters switch to $R$ without changing their legislative vote, they obtain $x^{R}$ with certainty. Hence $(I, z)$ is dominated.

2. $u\left(x^{R}, 1 / 2\right)<u\left(x^{D}, 1 / 2\right)$

Proof analogous to case 1.

3. $u\left(x^{R}, 1 / 2\right)=u\left(x^{D}, 1 / 2\right)$; this implies that $x^{R}>1 / 2>x^{D}$.

A. Assume that there are some voters with $\theta_{1}>1 / 2$ who vote $D$ for president under $z$. Since for these voters $u\left(x^{R}, \cdot\right)>u\left(x^{D}, \cdot\right),(I, z)$ can be dominated if these voters switch to $R$ for president.

B. Assume, therefore, that there are no voters with $\theta_{1}>1 / 2$ who vote $D$ for president under $z$. That is, $z$ must have a cutpoint $\hat{\theta}=1 / 2$.

1. Assume there are voters with $\theta_{1} \geq x^{R}$ who voted $R D$ under $z$. These voters do not satisfy condition (C) of Proposition 1. Hence, $(I, z)$ is dominated.

ii. Assume there are voters with $1 / 2<\theta_{1}<x^{R}$ who voted $R R$ under $z$. Then there must exist (a) a measurable interval $\delta=[\underline{\delta}, \bar{\delta}]$, with $1 / 2 \leq \underline{\delta}<\bar{\delta}<$ $x^{R}$ such that, when RR voters in $\delta$ switch their legislative votes to $D$, the outcomes become $x^{R^{\prime}}, x^{D^{\prime}}$, with $x^{D^{\prime}}<x^{D}<x^{R^{\prime}}<x^{R}$ and (b) an interva] [ $\left.\varepsilon, 1 / 2\right]$ 
such that all voters in this interval and $\delta$ prefer $x^{R^{\prime}}$ to both $x^{R}$ and $x^{D}$. Consequently, the voters in $S=[\varepsilon, 1 / 2] \cup[\delta]$ will be better off if voters in $[\varepsilon, 1 / 2]$ change their presidentlal vote to $R$ while voters in $\delta$ switch their legislative vote to $D$. Thus, $(I, z)$ is dominated. The construction is 11 lustrated in Figure $\mathrm{A}-1$.

iii) The remaining possiblity is that all voters in $\left(1 / 2, x^{R}\right)$ vote $R D$ under $z$ and all those above $x^{R}$ vote $R R$. But then domination can occur by shifting the presidential election to $D$ unless it is the case that all voters in $\left[0, x^{D}\right]$ vote $D D$ and all those in $\left(x^{D}, 1 / 2\right)$ vote $D R$. But in this last situation, it is direct to show that condition (C) cannot be satisfled.

Q.E.D

\section{Proof of Proposition 4 :}

\section{Non-Cutpoint Strategies}

We begin by amending the statement of the proposition to permit the use of non-cutpoint strategies.

Conditions [1.Ai] and [1.Biv] become

[1.A.i] $1-M_{R z}+\sigma(\{i: i$ votes $R$ for president under $z$ and $i \epsilon$ $\left.\left.\left(0, \tilde{\theta}_{R}\right)\right\}\right)>1 / 2$

[1.B.iv] $1-M_{\mathrm{Rz}}+\sigma(\{i: i$ votes $R$ for president under $z$ and $i \in$ $\left.\left.\left[0, \theta^{\prime}\right)\right\}\right)>1 / 2$.

In addition, for $\theta_{D}+k \stackrel{\sum}{=} \tilde{\theta}_{R^{\prime}}$ l to insure that $\tilde{\theta}_{R}$ is not dominated by creating a tied presidential racel there does not exist $x^{*}, x^{R}, \theta^{\prime \prime}$, which satisfy:
[1.v] $\quad x^{D}=\theta_{D}+k V_{R}^{T} ; x^{R}=\theta_{R}-k\left(1-V_{F}^{T}\right)$
[1.vi] $V_{R}^{I}=1-\tilde{\theta}_{R}+\theta^{\prime \prime}$
[1. vii] $(1 / 2)\left[u\left(x^{R}, \theta^{\prime \prime}\right)+u\left(x^{D}, \theta^{\prime \prime}\right)\right]=u\left(\tilde{\theta}_{R}, \theta^{\prime \prime}\right)$ 
[1.vi1i] $1-M_{R z}+\sigma(\{1: 1$ votes $R$ for president under $z$ and $\left.\left.i \in\left(0, \theta^{\prime \prime}\right)\right\}\right)=1 / 2$.

Proposition 3 establishes that ties cannot, be SN. Proposition 1 establishes that, when the presidential components of strategies result in an $R$ president, domination will occur unless the legislative component obeys $\tilde{\theta}_{R}$. Similarly, in the case of D president, we must have $\tilde{\theta}_{D}$. Therefore, the only possible SN outcomes correspond to $\tilde{\theta}_{R}$ and $\tilde{\theta}_{D^{\prime}}$. We focus on the case of $\tilde{\theta}_{R}$, the proof being similar for $\tilde{\theta}_{D^{*}}$.

First consider domination by strategies that result in a $D$ president and a policy $x^{*}$. To achleve dominance of $\tilde{\theta}_{R}$ we must have $x^{*}<\tilde{\theta}_{R}$. If $x^{*} \geq \tilde{\theta}_{R^{\prime}}$ since $x^{*}$ would require $V_{R}^{*}>1-\tilde{\theta}_{R^{\prime}}$ this implies that the dominating $S$ must contain voters with $\theta_{1}<\tilde{\theta}_{R}$ who do not obtain higher utility when $V_{R}^{*}>1-\tilde{\theta}_{R}$.

Assume 1.A. Consider a coalition $S=\left[0, \tilde{\theta}_{R}-\xi\right], \xi>0$. Since $k<1$, members of $\mathrm{S}$ can coordinate their legislative strategies in a manner such that $\tilde{\theta}_{R}>x^{*}>\tilde{\theta}_{R}-\xi$. Hence, if holding strategies of players not in $S$ constant, domination will occur if and only if $S$ has sufficient votes to elect D president, Condition [1.Ai] Identifies the largest possible S capable of electing $D$ president.

Assume 1.B. Assume the dominating pair $(S, y)$ leads to a policy $x^{*}<$ $\ddot{\theta}_{R^{\prime}}$ From (2), $x^{*}$ satisfies [1.B1]. By the assumptions on the utility function, there is a unique indifferent voter such that $\theta^{\prime}$ satisfies [1.B111]. Moreover, all voters $i$ such that $\theta_{1}<\theta^{\prime}$ prefer $x^{*}$ to $\tilde{\theta}_{R}$. Therefore, $S<\left[0, \theta^{\prime}\right]$ and [1.Biv] specifles whether $S$ is able to elect $D$ president. Moreover, if under $y$ the legislative vote required by [1.Bi] satisfies

$$
V_{R}^{*} \leq 1-\tilde{\theta}_{R}+\theta^{\prime}
$$


$S$ will have sufficient votes to bring about $x$ "when $D$ is elected. If this is the case, it is straightforward to show that there exist $x^{*+}, \theta^{+}$such that $\tilde{\theta}_{\mathbf{R}}$ is dominated and [1.Bii] is satisfled. Thus $\tilde{\theta}_{\mathbf{R}}$ cannot be dominated by shifting the vote to $D$ president if and only if [1.Bi] - [1.Biv] are not satisfied, .

Remark: If $\tilde{\theta}_{\mathrm{R}} \leq 1 / 2$ and $z$ is a presidential cutpoint strategy, condition [1.Biv] cannot be satisfied; therefore, all cutpoints $z$ with $\tilde{\theta}_{R} \leq$ $1 / 2$ cannot be dominated by shifting the presidency to $D$.

It remains to specify when $\tilde{\theta}_{R}$ can be dominated by a tie, which generates potential outcomes $x^{D}, x^{R}$. By an argument parallel to that used to show $x^{*}<$ $\tilde{\theta}_{R}$, we must have $x^{D}<\tilde{\theta}_{R}$. Now if $x^{D}<\tilde{\theta}_{R}$, by the assumption that all voters have identically "shaped", concave $u(\cdot)$, we must have $x^{D}<\tilde{\theta}_{R} \leq x^{R}$ and $\theta^{\prime \prime}<$ $\tilde{\theta}_{R}$, with $\theta^{\prime \prime}$ the unique type indifferent between the lottery and $\tilde{\theta}_{R}$.

Conditions [1.v] - [1.viii] specify a coalition $S=\left[0, \theta^{\prime \prime}\right.$ ) with exactly enough $R$ votes for president under $z$ to create a tie with $\theta^{\prime \prime}$ the indifferent voter. It is direct to show that $\theta^{\prime \prime} \leq \theta^{\prime}$. (See Flgure $A-2$, where $x^{\text {"R }}$ denotes the outcome where a tie occurs with $x^{*}$ the outcome when $D$ wins the lottery for president.) Therefore, it is redundant to replace the equality in [1.viii] with an inequality. In the case of a strict inequality, domination can occur via a shift to $D$ president with the outcome $x$ *.

Q.E.D.

\section{Proof of Proposition 5}

Characterization of an abstract stable set

A set $K$ exists and consists of all the pairs $(S, z) \quad S \subseteq I$ that satisfy:

1. The strategy $z$ implies $R$ is president with vote $M_{R z}>1 / 2$; the legislative component of $z$ satisfies $(C)$ for members of $S$ and implies $V_{B}$ and a policy of 
$x$ and there does not exist $\theta^{\prime}, x^{*}, v_{R}^{*}$ such that:

$$
\begin{aligned}
& \text { [1.Ai] } x^{*}=\theta_{D}+k_{R}^{*} \\
& \text { [1.Aii] } v_{R}^{*}=v_{R}+\sigma\left(\left\{1: i \in S \text { and } x^{*} \leq \theta_{1}<\theta^{\prime}\right\}\right) \\
& \text { [1.Aii] } u\left(x^{*}, \theta^{\prime}\right)=u\left(x, \theta^{\prime}\right) \\
& \text { [1.Aiv] } 1-M_{R z}+\sigma(\{i: i \in S \text { and } i \text { votes } R \text { for president under } z \\
& \text { and } \left.\left.i \in\left[0, \theta^{\prime}\right)\right\}\right)>1 / 2 .
\end{aligned}
$$

and that there does not exist $\theta^{\prime \prime}$ and $T \leq S \leq\left(0, \theta^{\prime \prime}\right)$ such that

$$
\begin{aligned}
& \text { [1.Biil] } u\left(x, \theta^{\prime \prime}\right)=u\left(\theta_{D}+k V_{R^{\prime}}, \theta^{\prime \prime}\right) \\
& \text { [1.Biv] } 1-M_{R z}+\sigma(\{1: i \in T \text { and } i \text { votes } R \text { for president under } \\
& z\})=1 / 2 .
\end{aligned}
$$

2. The strategy $z$ implies $D$ is president with vote $M_{R z}<1 / 2$; the legislative component of $z$ satisfies (C) for members of $S$ and implies a policy of $x$ and $z$ satisfies conditions symmetric to those of 1 .

3. The strategy $z$ implies a tie for president with vote $M_{R z}=1 / 2$; the legislative component of $z$ satisfies (C) for members of SCI, and implies $V_{R}$ and a lottery between policies $x^{R}$ and $x^{D}$, and there does not exist $\theta^{\prime \prime}, x^{*}, V_{R}^{T}$ such that either:

\section{A}

$$
\begin{aligned}
& \text { [3. Av] } x^{*}=\theta_{D}+k v_{R}^{T} \\
& \text { [3. Avi } v_{R}^{T}=v_{R}+\sigma\left(\left\{i: i \in S \text { and } x^{*} \leq \theta_{1}<\theta^{\prime \prime}\right\}\right) \\
& \text { [3. Avil] } u\left(x^{*}, \theta^{\prime \prime}\right)=(1 / 2)\left[u\left(x^{R}, \theta^{\prime \prime}\right)+u\left(x^{D}, \theta^{\prime \prime}\right)\right] \\
& \text { [3. Avili] } \sigma(\{i: i \in S \text { and } i \text { votes } R \text { for president under } z \text { and } i \in \\
& \left.\left.\left[0, \theta^{\prime \prime}\right)\right\}\right)>0 .
\end{aligned}
$$

or

3. B

$$
\text { [3. Bv] } \quad x^{*}=\theta_{R}-k\left(1-v_{R}^{T}\right)
$$


[3. Bvi] $V_{R}^{T}=V_{R}-\sigma\left(\left\{i: i \in S\right.\right.$ and $\left.\left.x^{*} \geq \theta_{1}>\theta^{\prime \prime}\right\}\right)$

[3. Bvii] identical to [3, Avii]

[3. Bviii] $\sigma\left(\left\{i\right.\right.$ : $i \in S$ and $I$ votes $D$ for president under $z$ and $\left.\left.\left.i \in\left(\theta^{\prime \prime}, 1\right]\right)\right\}\right)$ $>0$.

\section{Existence}

If the median voter does not strictly prefer $\tilde{\theta}_{D}$ to $\tilde{\theta}_{R}$ it is direct to show that $\left(I, \tilde{\theta}_{R}\right)$ satisfies (1). Likewise, if the median voter does not strictly prefer $\tilde{\theta}_{R}$ to $\tilde{\theta}_{D^{\prime}},\left(I, \tilde{\theta}_{D}\right)$ satisfies (2). Hence, the proposed $K$ is non-empty.

\section{External Stability}

First consider $(S, z)$ where (C) is not satisfied. By Proposition 1 there exists $(T, y)$ such that $(S, z)<(T, y)$. Now consider $M_{R z} \neq 1 / 2$. It is possible to find a dominating $(T, y) \in K$ because $\sigma(T)$ can be sufficiently small that $I$ is not pivotal in the presidential election. If $M_{R z}=1,2$, either $(T, y)$ satisfies (3) or, if it does not, there exists $T^{\prime} \subset T$, with $\sigma\left(T^{\prime}\right)$ arbitrarily small, where presidential as well as legislative components of strategies are shifted and $(S, z)<\left(T^{\prime}, y^{\prime}\right)$ and $\left(T^{\prime}, y^{\prime}\right) \in K$ since $\left(T^{\prime}, y^{\prime}\right)$ satisfies (C) and $I^{\prime}$ is not pivotal in the presidential elections.

Consider next (C) satisfied [1.Ai] - [1.Aiv] are satisfied and therefore $(S, z) \notin K$. Let $T=\left\{i \in S\right.$ and $\left.i \in\left[0, \theta^{\prime}\right)\right\}$. In the dominating strategy $y$, members of $T$ in $\left[0, x^{*}\right]$ use $D D$ and members of $T$ in $\left(x^{*}, \theta^{\prime}\right)$ use DR. By the construction, $D$ is elected president under $y$ and members of $T$ receive higher utility under $y$ than under $z$. Hence, $(S, z)<(T, y)$. Moreover, $(T, y)$ trivially satisfies ( 2$)$. Hence $(T, y) \in K$.

Consider next (C) satisfied, [1.Ai] - [1.Aiv] not satisfied, but 
[1.Biil] - [1.Biv] satisfied. For $T$ in the construction, let the member of $T$ deviate to $y$ continue to vote $D$ in the legislative election in order to satisfy (C). By construction, no $T^{\prime} \in I$ can elect a $D$ president. It is also direct to show that members of $T$ would be worse off by switching back to $R$ president. Hence, $(T, y)$ satisfies (3). Hence $(T, y) \in K$.

A similar argument applies when $D$ is elected president and (2) is not satisfied.

Finally, consider the case of a tie for president and (C) satisfied but either [3.Av] - [3.Aviii] or [3.Bv] - [3. Bviii] satisfied. Again, using an appropriate subset of voters in $S$, it is possible to construct a dominating $(T, y)$ that belongs to $K$.

\section{Internal Stability}

Given that (C) is satisfied, members of $K$ are obviously stable against other members of $K$ that result in the same presidential outcome. The construction used in ( 3 ) guarantees that presidential ties in $K$ cannot be upset by shifting to a certain winner. Similary, the construction of (1) and (2) guarantee that certain winners in $\mathrm{K}$ cannot be dominated by members of $\mathrm{K}$ where the other party wins the presidency. It remains to demonstrate, without loss of generality, that a member of $K$ satisfying (1) cannot be dominated by a tie that satisfies (C) other than ties that satisfy [1.Biii][1.Biv]. Let $(S, z)$ satisfying (1) be upset by a tie that satisfies (C). Then a voter $\theta^{\prime \prime}$ is indifferent between a lottery and $x$. By arguments parallel to chose used in pruving Proposition 4, the policy $x^{D}$ in the lottery must be less than $x$ and the coalition that creates the tie can include only voters in $\left\{\theta, \theta^{\prime}\right)$. Moreover, $x^{R} \geq x$ or else the voter is not indifferent. Therefore, all $\theta_{1}<\theta^{\prime}$ strictly prefer $x^{D}$ to $x^{R}$. Consequently, to satisfy (C) in the 
tie, all defectors must continue to vote $D$ in the legislative election. But then $x^{R}=x$. So if $(S, z)$ is dominated by a tie that satisfles (C), [1.Biii] - [1.Biv] must be satisfled. Consequently, $K$ is internally stable.

\section{$(I, z)$ in the Stable Set}

Finally, if $S=I$ and given a sure victory in the presidential election, the cutpoints for the legislative election are either $\tilde{\theta}_{R}$ or $\tilde{\theta}_{D}$ by condition (C) .

We complete the proof by showing that all $(I, z)$ representing $t$ ied outcomes that satisfy (C) are dominated by members of $X$. Consider cases (1), (2) and (3A) of the proof to Proposition 3. Since $\sigma(T)$ may be made arbitrarally small, it is clearly possible to construct a dominating (T,y) that is a member of $K$ for $R$ winning the presidency. The construction of the dominating coalition in $(3 B-i i)$ also generates a member of $\mathrm{K}$. Members of the dominating coalition prefer $x^{R^{\prime}}$ to $x^{D^{\prime}}$ and cannot obtain a more moderate outcome than $x^{D}$ by shifting the outcome to D's winning the presidency. The remaining cases covered by Proposition 3 involve situations where (C) is not satisfied.

Q.E.D.

\section{Proof of Proposition 7 .}

The key to the proof is that the realization of a only affects the relative proportions of voters with weakly dominant strategies of voting $R$ and $D$ as a result of $(26)$.

Note that for a given strategy $z$ (28) can be written in terms of the derived density $\phi\left(V_{R}\right)$ of $V_{R}$ as

$$
E u_{1}^{R}=\int_{V_{R}}^{\bar{V}_{R}}-\frac{1}{2}\left[\theta_{R}-k\left(1-V_{R}\right)-\theta_{1}\right]^{2} \phi\left(V_{R}\right) d V_{R}
$$


where $\bar{V}_{R}=1+w-\theta_{R}+\sigma_{R}$ and $\underline{V}_{R}=1-w-\theta_{R}+\sigma_{R}$ and $\sigma_{R}=\dot{\sigma}\left\{1: \theta_{R}>\theta_{D}\right.$ and 1 votes $R$ under $z$ \} since voters $\theta_{1} \leq \theta_{D}$ with weakly dominant strategies always vote $D$ and $\theta_{1} \geq \theta_{R}$ always vote $R$.

Since $E u_{i}^{R}$ is quadratic in $V_{R}$, $E u_{1}^{R}$ can be expressed as a function of the mean and varlance of $V_{R}$. It is readlly shown that the variance of $V_{R}$ is a constant for all strategies $z$ where voters employ weakly dominant strategies. Thus, voter preferences between alternative strategies depend only on $E_{R}$. By an argument analogous to the proof of Lemma 1, it can be shown that preferences are single-peaked in $E_{R}$. Proposition 7 then follows by arguments directly parallel to the proofs of Proposition 1 and 2.

\section{Proof of Proposition 9 .}

We develop the proof using a restrictive assumption in addition to $A 1$. A3. Strategies $z$ are restricted to be cutpolnt strategies.

Without this restriction, the proof requires a construction similar to that used for Proposition 5.

\section{Characterization of an abstract stable set.}

The abstract stable set $K$ consists of all pairs $(S, z), S \subseteq$ I such that efther, given $z, C^{1}$ and $C^{p}$ are satisfied for all $i \in S$ in the first period or, If any of these conditions are not satisfied for $z$, $S$ does not contain the cutpoints in $z$ that correspond to the conditions that are violated. In addition, the second period cutpoints are $\tilde{\theta}_{D 2}^{*}, \tilde{\theta}_{R 2}^{*}$

Remark. It is direct to show that the unique member of $K$ of the form $\left(I_{w}, z\right)$ has $z=z^{*} \equiv\left(\hat{\theta}^{*}, \tilde{\theta}^{*}, \tilde{\theta}_{\mathrm{D} 2}^{*}, \tilde{\theta}_{\mathrm{R2}}^{*}\right)$.

\section{External Stability.}

Assume that $(S, z) \notin K$ and that there exists a cutpoint $\zeta$ contained in $S$ for which the approprlate $C$ condition is not satisfied for one or more (if 
two or more contests share an identical cutpoint) contests. Then there exists a Coalition $T$ either of the form $[\zeta-\varepsilon, \zeta]$ or $[\zeta, \zeta+\varepsilon](\varepsilon>0)$ such that all $i \in T$ vlolate the $C$ condition for one or more contests referenced by $\zeta$. Moreover $\sigma(T)$ can always be made sufficiently small such that, when members of $T$ reverse their $z$ votes for these contests, leading to some $y$, $T$ satisfies the appropriate $C$ conditions given $y$ and $T$ contains no other cutpoint. Hence, $(T, y) \in K$.

\section{Internal Stability}

Without loss of generality, assume $\hat{\theta}^{*} \leq \tilde{\theta}^{*}$, the opposite case being symmetric.

1. If $(S, z) \in K$ and no cutpoint in $z$ is in $S$ or in a boundary of $S$, $(S, z)$ is internally stable by $A 3$. (Note: if one boundary of $S$ is open but the cutpoint is on the boundary, some $T$ in $S$ can still defect to another cutpoint strategy y.)

2. A3, Lemma 2 and Lemma 4 imply that $(S, z) \in K$ is internally stable if $(S, z)$ or its boundaries does not contain both $\hat{\boldsymbol{\theta}}$ and $\tilde{\theta}$.

3. $\mathbf{A} 3$ and Lemma 2 and Lemma 4 further imply that if $(S, z) \in K$ and $(S, z)<$ $(T, y)$ where $z=\left(\hat{\theta}^{z}, \tilde{\theta}^{x}, \tilde{\theta}_{D Z^{\prime}}^{*}, \tilde{\theta}_{R 2}^{*}\right)$ and $y=\left(\hat{\theta}^{\prime}, \tilde{\theta}^{\prime}, \tilde{\theta}_{D Z^{\prime}}^{\prime *} \tilde{\theta}^{\prime}{ }^{*}\right)$, a necessary condition is that $\hat{\theta}^{\prime} \neq \hat{\theta}^{z}, \tilde{\theta}^{\prime} \neq \tilde{\theta}^{z}$. This means that $y$ must differ from $z$ in both $t=1$ components.

4. Assume, therefore, that the necessary condition is satisfied. We first show that all (S, $z^{*}$ ) are internally stable. By A3, any dominating $T$ must include the half-open intervals defined by $\hat{\theta}^{*}$ and $\tilde{\theta}^{\prime}$ and $\tilde{\theta}^{*}$ and $\tilde{\theta}^{\prime}$. But the stability property of $\mathbf{A 2}$ can be used to establish that either $\tilde{\theta}\left(\hat{\theta}^{\prime}\right) c$ : $\hat{\theta}\left(\tilde{\theta}^{\prime}\right)$ (or both) is in the direction of the * cutpoint from the ' cutpoint. Hence, the $C$ conditions are not satisfied on $T$, implying that ( $S, z^{*}$ ) is not. 
dominated by a member of $K$.

5. Next consider $(S, z) \in K, z \neq z^{*}$. If $(S, z) \in K$, it is direct to show that both $t=1$ cutpoints cannot be strictly interior to $S$.

Assume both cutpoints are on a boundary of $S$. Case a. Assume domination occurs with $\left.\hat{\theta}^{\prime}\right\rangle \hat{\theta}^{z}$. But then $\tilde{\theta}^{\prime}\left\langle\tilde{\theta}^{z}\right.$ or members of $S$ in a neighborhood of $\hat{\theta}^{z}$ will not be strictly better off with $(T, y)$. So $\left(\hat{\theta}^{z}, \hat{\theta}^{\prime}\right) \cup$ $\left(\tilde{\theta}^{\prime}, \tilde{\theta}^{z}\right) \subseteq \mathrm{T} \subset \mathrm{S}$. Moreover, $C^{\mathrm{P}}$ on $\mathrm{S} \Rightarrow \hat{\theta}\left(\tilde{\theta}^{z}\right) \leq \tilde{\theta}^{\mathbf{z}}$ and $C^{1}$ on $S \Rightarrow \tilde{\theta}\left(\hat{\theta}^{z}\right) \geq \tilde{\theta}^{z}$. Since $z \neq z^{*}$, at least one of the inequalities must be strict. It is therefore direct to show, using the stability property of 12 , that either $C^{1}$ or $C^{P}$ is not satisfied for $(T, y)$. Hence, $(T, y) \notin X$. Case $b$. Assume therefore that domination is achieved with $\hat{\theta}^{\prime}<\hat{\theta}_{z}$. Proof of non-domination is parallel to Case a. Therefore, if $(S, z) \in X$ and no $t=1$ cutpoint is strictly interior to $S,(S, z)$ is internally stable.

Finally assume one cutpoint is interior and the other is not. Without loss of generality, assume the interior cutpoint is $\tilde{\theta}^{z}$. For $S$ to satisfy $C^{l}$, it thus must be the case that $\tilde{\theta}^{z}=\tilde{\theta}\left(\hat{\theta}^{z}\right)$. Therefore, $\hat{\theta}^{z} \neq \hat{\theta}(\tilde{\theta})$ from the assumption that $z \neq z^{*}$. This fact permits proof of non-domination in a manner parallel to the preceeding paragraph.

Thus, all $(S, z) \in K$ are internally stable.

Q.E.D. 


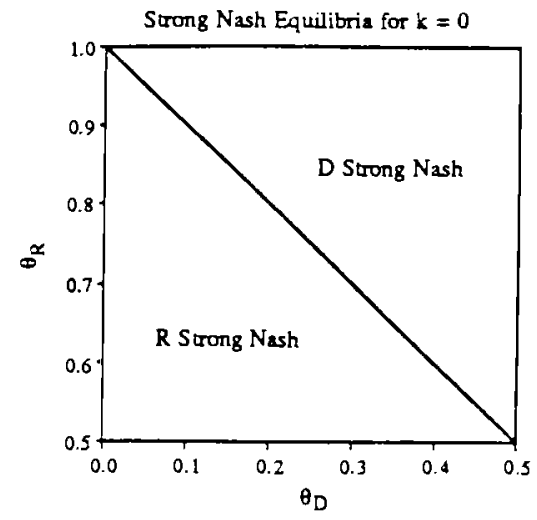

Figure 1-A

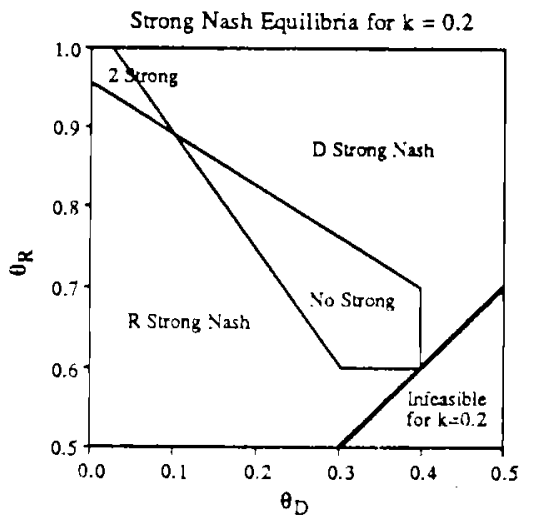

Figure 1-B

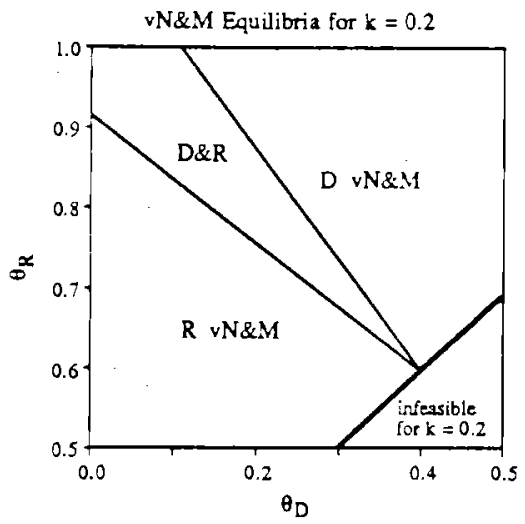

Figure 1-D

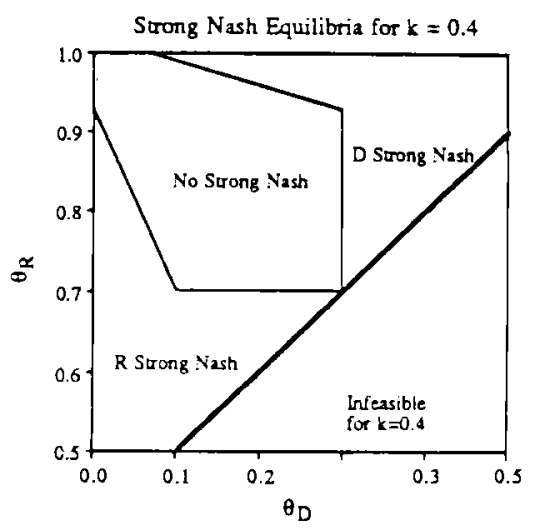

Figure 1-C

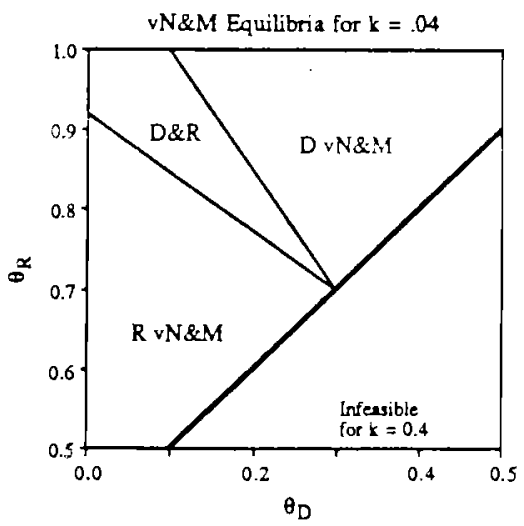

Figure 1-E 


\section{Figure 2}

\section{The Strong Nash Dominating Coalition}

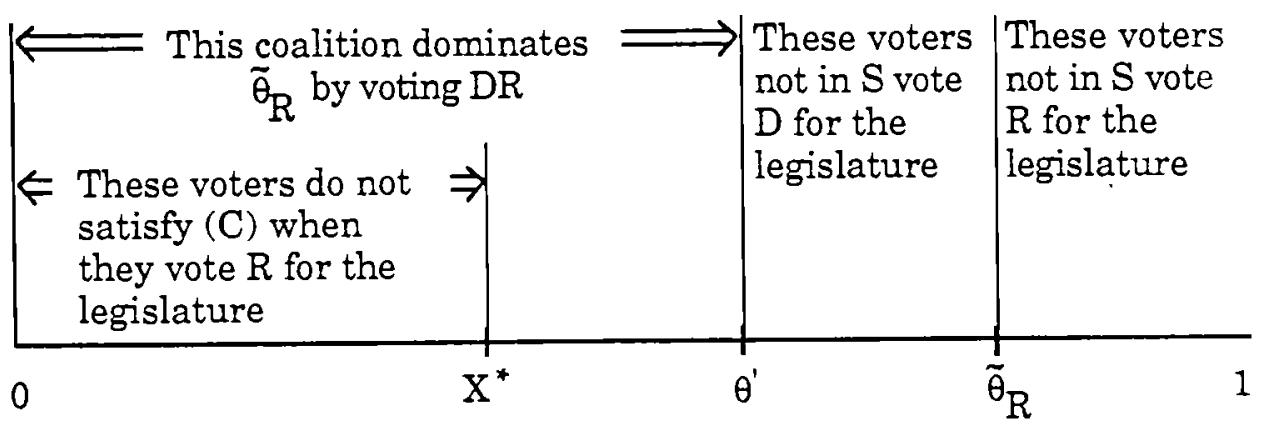

\section{The vN\&M Dorinating Coalition}

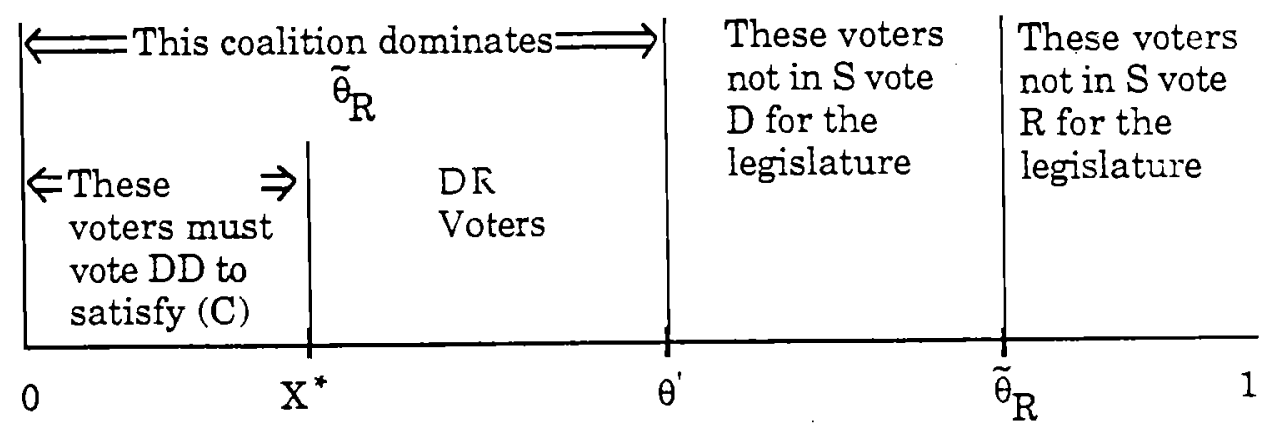


Figure 3

Equilibrium with Incomplete Information

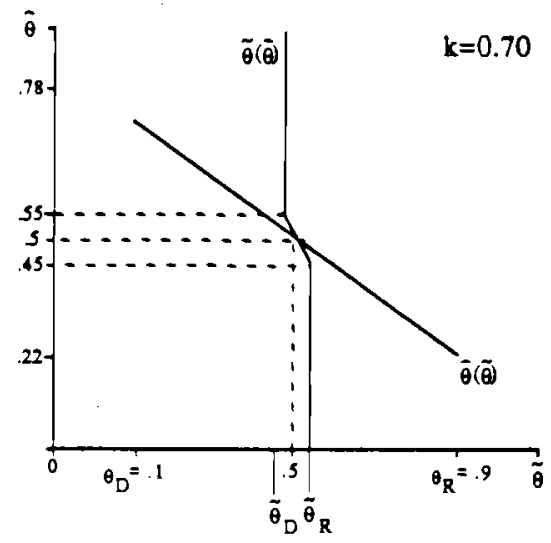

$3 \cdot A$

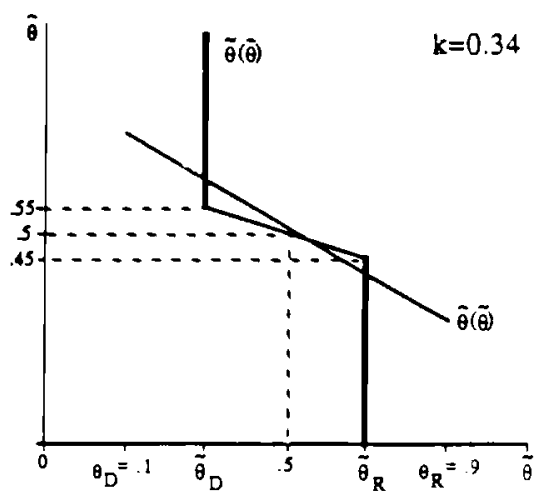

$3 \cdot B$

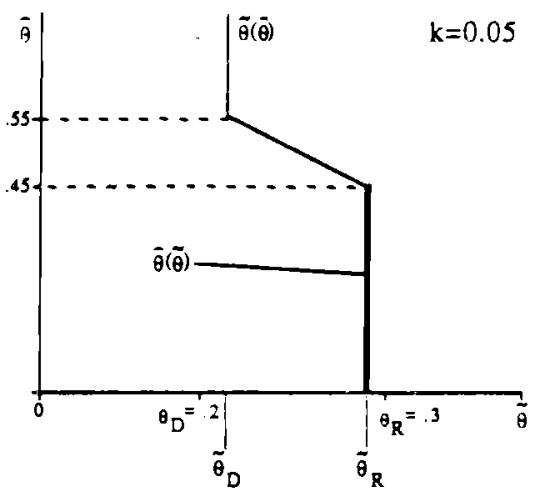

3-C

Notes: $w=0.05$ in all panels.

Heavy lines denote equilibria with Presidential winner certain. 


\section{Figure A-1}

\section{Domination of a Tie When Median Voter is Indifferent at Tie}

DD

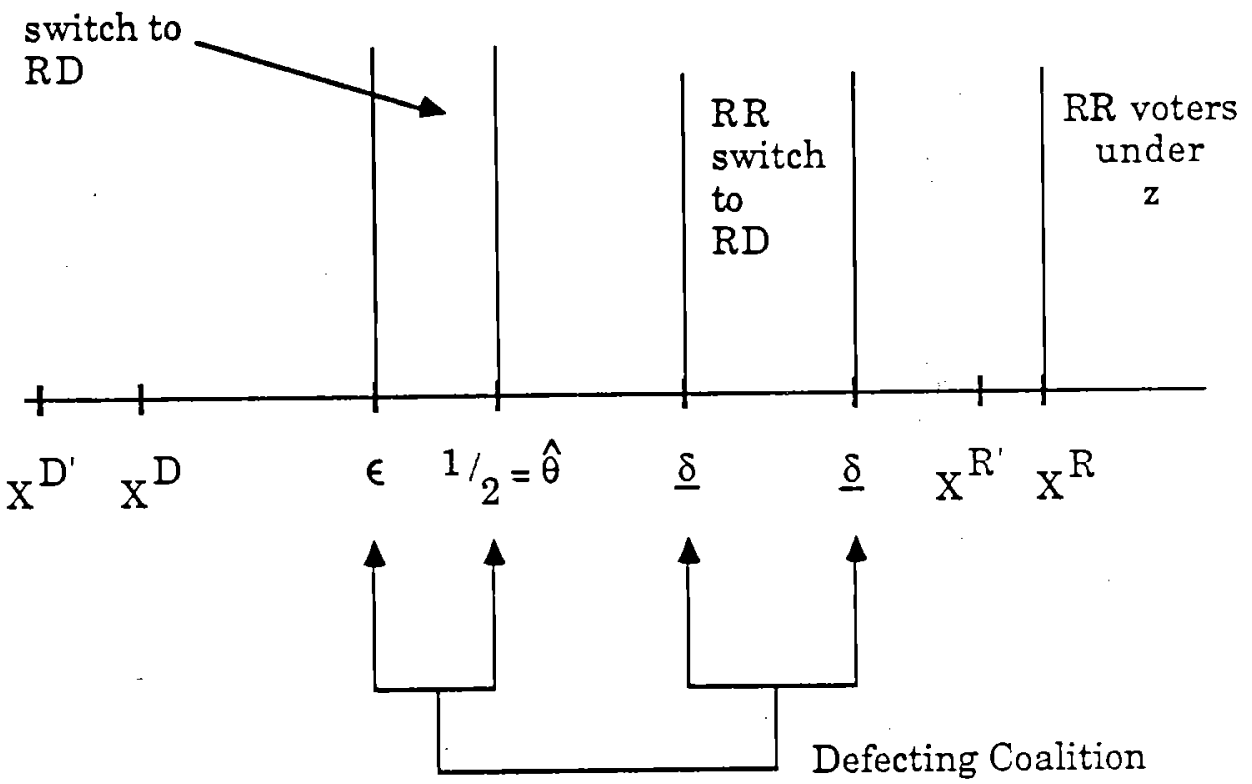




\section{Figure A-2}

\section{When Domination Occurs Via a Tie}

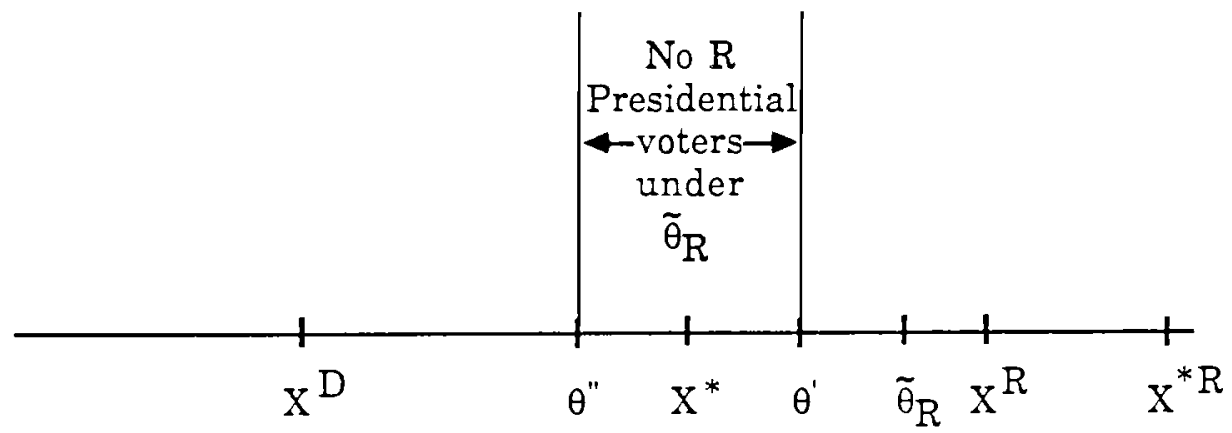

$\theta^{\prime} \quad$ - indifferent voter if D President and policy $=X^{*}$

$\theta^{\prime \prime} \quad$ - indifferent voter if tie and policies $\mathrm{X}^{\mathrm{D}}, \mathrm{x}^{\mathrm{R}}$

$X^{*} R$ - policy if $R$ wins given tie vote for President and legislative vote equal to vote when D President and policy is $\mathrm{X}^{*}$

$\tilde{\theta}_{\mathrm{R}} \quad$ - proposed equilibrium outcome, $R$ President 